\title{
Sojourn Times in Fluid Queues with Independent and Dependent Input and Output Processes ${ }^{\text {ts }}$
}

\author{
Gábor Horváth ${ }^{\mathrm{a}, \mathrm{c}}$, Miklós Telek ${ }^{\mathrm{a}, \mathrm{b}}$ \\ ${ }^{a}$ Budapest University of Technology and Economics, Department of Networked Systems and Services \\ ${ }^{b} M T A-B M E$ Information Systems Research Group \\ ${ }^{c}$ Inter-University Center of Telecommunications and Informatics, Debrecen
}

\begin{abstract}
Markov Fluid Queues (MFQs) are the continuous counterparts of quasi birth-death processes, where infinitesimally small jobs (fluid drops) are arriving and are being served according to rates modulated by a continuous time Markov chain. The fluid drops are served according to the First-Come-First-Served (FCFS) discipline. The queue length process of MFQs can be analyzed by efficient numerical methods developed for Markovian fluid models. In this paper, however, we are focusing on the sojourn time distribution of the fluid drops.

In the first part of the paper we derive the phase-type representation of the sojourn time when the input and output process of the queue are dependent. In the second part we investigate the case when the input and output processes are independent. Based on the age process analysis of the fluid drops, we provide smaller phase-type representations for the sojourn time than the one for dependent input and output processes.
\end{abstract}

Keywords: Markov fluid model, Age process, Phase type representation.

\section{Introduction}

Two different types of queueing systems can be distinguished based on the nature of the jobs the queue is operating on. There are discrete queues where jobs have a finite size (and queue length is measured in number of jobs), and continuous queues where the size of the jobs is infinitesimally small (and queue length is measured in amount of jobs $)^{1}$. The research on discrete queues [1] have commonly preceded the one on their continuous counterparts [2].

A class of queues which turned out to be particularly useful in the modeling practice are queues where the arrivals and services are modulated by a background Markov chain. A lot of results are available for such systems in case of discrete jobs: elegant and numerically efficient procedures to obtain the matric-geometric distribution of the queue length [3] and the matrix-exponential distribution of the sojourn time [4]. The introduction of the age process based analysis of discrete queues $[5,6,7]$ made it possible to obtain a lower order matrix-exponential representation of the sojourn time distribution if the arrival and service processes are independent.

The matrix-analytic approach to solve the discrete queues has been ported to continuous queues several decades later [8]. However, the efficient representation of the sojourn time distribution of continuous queues

\footnotetext{
This work was supported by the Hungarian research project OTKA K101150, by the European Union (co-financed by the European Social Fund) through the TAMOP-4.2.2C-11/1/KONV-2012-0001 project, and by the János Bolyai Research Scholarship of the Hungarian Academy of Sciences.

Email addresses: ghorvath@hit.bme.hu (Gábor Horváth), telek@hit.bme.hu (Miklós Telek)

${ }^{1}$ The "discrete" and "continuous" classification used in this paper has nothing to do with "discrete time" and "continuous time" stochastic processes. We deal with continuous time processes throughout the paper.
} 
are not known yet. This paper focuses on the solution of this problem and is organized as follows. We introduce Markov fluid queues in Section 2 and discuss their sojourn time analysis with dependent input and output processes in Section 3. This general result is specified for the case of independent input and output processes in Section 4, which allows a much smaller phase type representation of the sojourn time distribution.

\section{Markov Fluid Queues}

Markov fluid queues (MFQs) are characterized by a two-dimensional Markov process $\{\mathcal{X}(t), \mathcal{Z}(t), t>0\}$, where $\mathcal{X}(t)$ represents the fluid level and $\mathcal{Z}(t)$ is the underlying continuous time Markov chain with state space $\mathcal{S}$ of size $|\mathcal{S}|=N$ and generator matrix $\mathbf{Q}$ that modulates the rate at which fluid enters and leaves the queue.

The rate of the fluid entering the queue in state $i$ of the background process is denoted by $r_{i}^{(i n)}$. The diagonal matrix $\mathbf{R}_{\text {in }}$ is composed by fluid rates $r_{i}^{(i n)}, i=1, \ldots, N$. The rate at which the fluid can leave the buffer in state $i$ is denoted by $r_{i}^{\text {(out) }}$, with the corresponding diagonal matrix denoted by $\mathbf{R}_{\text {out }}$. The overall fluid rate determining the rate at which the level of the queue changes is the difference between the input and the output rates, $\mathbf{R}=\mathbf{R}_{\mathbf{i n}}-\mathbf{R}_{\text {out }}$. This system is the continuous equivalent of the quasi birth death (QBD) queue. In a $\mathrm{QBD}$ queue there is a background process where marked transitions generate an arrival or a service of a discrete job. Due to the common background process the arrivals and services are dependent. In case of the MFQs the jobs (fluid drops) are infinitesimally small, but their input and output rates are also dependent due to the common background process $\mathcal{Z}(t)$.

Formally, the behavior of the fluid buffer is as follows,

$$
\frac{d}{d t} \mathcal{X}(t)= \begin{cases}r_{\mathcal{Z}(t)}^{(\text {in })}-r_{\mathcal{Z}(t)}^{(\text {out })}, & \text { if } \mathcal{X}(t)>0 \\ \max \left\{0, r_{\mathcal{Z}(t)}^{(\text {in })}-r_{\mathcal{Z}(t)}^{(\text {out })}\right\}, & \text { if } \mathcal{X}(t)=0\end{cases}
$$

Let us denote the row vector of the transient state probabilities of the underlying CTMC for $t>0$ by $\xi(t)=\left\{\xi_{i}(t), i \in \mathcal{S}\right\}$ with $\xi_{i}(t)=P(\mathcal{Z}(t)=i)$, its stationary limit by $\xi$, the row vector of the stationary distribution of the fluid levels for $x>0$ by $\pi(x)=\left\{\pi_{i}(x), i \in \mathcal{S}\right\}$ with $\pi_{i}(x)=\lim _{t \rightarrow \infty} \lim _{\Delta \rightarrow 0}(1 / \Delta) P(\mathcal{X}(t) \in$ $(x, x+\Delta), \mathcal{Z}(t)=i)$, and the row vector of the stationary probabilities of empty buffer by $p=\left\{p_{i}, i \in \mathcal{S}\right\}$ with $p_{i}=\lim _{t \rightarrow \infty} P(\mathcal{Z}(t)=i, \mathcal{X}(t)=0)$.

$\pi(x)$ and $p$ are the solutions of the matrix differential equation [9]

$$
\frac{d}{d x} \pi(x) \mathbf{R}=\pi(x) \mathbf{Q}
$$

with boundary conditions

$$
\begin{aligned}
\pi(0) \mathbf{R} & =p \mathbf{Q}^{(\mathbf{0})} \\
p_{i} & =0, \quad \forall i: r_{i}=r_{i}^{(\text {in })}-r_{i}^{(\text {out })}>0
\end{aligned}
$$

and normalizing condition

$$
p \mathbb{1}+\int_{0}^{\infty} \pi(x) \mathbb{1} d x=1,
$$

where $\mathbb{1}$ is the column vector of ones with proper dimension defined by the context.

In (3) we have considered the case when the generator of the background process when the buffer is empty (denoted by $\mathbf{Q}^{(\mathbf{0})}$ ) can be different from the one when the buffer is non-empty (denoted by $\mathbf{Q}$ ). The cases when $\mathbf{Q}^{(\mathbf{0})}=\mathbf{Q}$ and $\mathbf{Q}^{(\mathbf{0})} \neq \mathbf{Q}$ will be referred to as "regular" and "irregular" boundary, respectively, in the sequel. ${ }^{2}$

\footnotetext{
${ }^{2}$ Fluid models are usually introduced with a regular boundary behavior. In this paper we need the more general definition, since several queueing models with practical relevance have an irregular behavior. We will discuss such fluid queueing models in later sections. Note however, that this is not a fluid queue specific issue. Simple discrete queues, like the MAP/MAP/1 queue has an irregular boundary behavior as well, since the service process freezes when the queue is idle.
} 
In the recent decades efficient numerical techniques were developed for the stationary queue length analysis of MFQs. E.g., it has been recognized that the matrix-analytic approach basing the efficient analysis of quasi birth-death processes (QBDs) can be applied to fluid models as well, making it possible to solve fluid models with a large number of states (up to several thousand) in a numerically stable way (see [8],[10]). Here we summarize the main steps of this approach. The matrix-analytic solution starts with partitioning the state space according to the associated rates to three sets $\mathcal{S}_{+}=\left\{i \in \mathcal{S}, r_{i}>0\right\}, \mathcal{S}_{-}=\left\{i \in \mathcal{S}, r_{i}<0\right\}$ and $\mathcal{S}_{0}=\left\{i \in \mathcal{S}, r_{i}=0\right\}\left(N_{+}=\left|\mathcal{S}_{+}\right|, N_{-}=\left|\mathcal{S}_{-}\right|, N_{0}=\left|\mathcal{S}_{0}\right|\right)$. Technically, the state partitioning can be achieved by a transformation with an appropriate permutation matrix $\mathbf{P}$ such that ${ }^{3}$

$$
\mathbf{P Q P}^{-1}=\left[\begin{array}{lll}
\mathbf{Q}_{++} & \mathbf{Q}_{+-} & \mathbf{Q}_{+0} \\
\mathbf{Q}_{-+} & \mathbf{Q}_{--} & \mathbf{Q}_{-\mathbf{0}} \\
\mathbf{Q}_{\mathbf{0}+} & \mathbf{Q}_{\mathbf{0}} & \mathbf{Q}_{\mathbf{0 0}}
\end{array}\right], \quad \mathbf{P R P}^{-1}=\left[\begin{array}{lll}
\mathbf{R}_{+} & & \\
& \mathbf{R}_{-} & \\
& & \mathbf{0}
\end{array}\right]
$$

where the entries of $\mathbf{R}_{+}$are all positive and the ones of $\mathbf{R}_{-}$are all negative.

The analysis is based on two important matrices, matrix $\boldsymbol{\Psi}$ and $\mathbf{K}$. Matrix $\boldsymbol{\Psi}$ has an simple probabilistic interpretation, entry $(\boldsymbol{\Psi})_{i, j}, i \in \mathcal{S}_{+}, j \in \mathcal{S}_{-}$is the probability that the background process is in state $j$ when the fluid level returns to 0 given that it was in state $i$ when the busy period (a non-empty period of the fluid queue) was initiated. Matrix $\boldsymbol{\Psi}$ is the solution to the algebraic Riccati equation

$$
\boldsymbol{\Psi}\left|\mathbf{R}_{-}\right|^{-1} \mathbf{Q}_{-+}^{\bullet} \Psi+\Psi\left|\mathbf{R}_{-}\right|^{-1} \mathbf{Q}_{--}^{\bullet}+\mathbf{R}_{+}{ }^{-1} \mathbf{Q}_{++}^{\bullet} \Psi+\mathbf{R}_{+}{ }^{-1} \mathbf{Q}_{+-}^{\bullet}=\mathbf{0}
$$

where matrix $\mathbf{Q}^{\bullet}$ is the generator of the Markov chain from which the states with zero rates are censored out, thus

$$
\mathbf{Q}^{\bullet}=\left[\begin{array}{ll}
\mathbf{Q}_{++}^{\bullet} & \mathbf{Q}_{+-}^{\bullet} \\
\mathbf{Q}_{-+}^{\bullet} & \mathbf{Q}_{--}^{\bullet}
\end{array}\right]=\left[\begin{array}{ll}
\mathbf{Q}_{++} & \mathbf{Q}_{+-} \\
\mathbf{Q}_{-+} & \mathbf{Q}_{--}
\end{array}\right]+\left[\begin{array}{l}
\mathbf{Q}_{+\mathbf{0}} \\
\mathbf{Q}_{-\mathbf{0}}
\end{array}\right]\left(-\mathbf{Q}_{\mathbf{0 0}}\right)^{-1}\left[\begin{array}{ll}
\mathbf{Q}_{\mathbf{0}+} & \mathbf{Q}_{\mathbf{0}-}
\end{array}\right]
$$

If the drift is negative, i.e. $\xi \mathbf{R}_{\mathbf{i n}} \mathbb{1}<\xi \mathbf{R}_{\text {out }} \mathbb{1}$, all eigenvalues of matrix $\mathbf{K}$ have negative real parts (consequently has full rank and invertible) and can be expressed from $\boldsymbol{\Psi}$ as

$$
\mathbf{K}=\mathbf{R}_{+}{ }^{-1} \mathbf{Q}_{++}^{\bullet}+\Psi\left|\mathbf{R}_{-}\right|^{-1} \mathbf{Q}_{-+}^{\bullet}
$$

Furthermore, we introduce matrix $\mathbf{B}$ as

$$
\mathbf{B}=\left[\begin{array}{ll}
\mathbf{I} & \boldsymbol{\Psi}
\end{array}\right]\left[\begin{array}{cc}
\mathbf{R}_{+} & \\
& \left|\mathbf{R}_{-}\right|
\end{array}\right]^{-1}\left[\begin{array}{lll}
\mathbf{I} & \mathbf{0} & \mathbf{Q}_{+\mathbf{0}}\left(-\mathbf{Q}_{\mathbf{0 0}}\right)^{-1} \\
\mathbf{0} & \mathbf{I} & \mathbf{Q}_{-\mathbf{0}}\left(-\mathbf{Q}_{\mathbf{0 0}}\right)^{-1}
\end{array}\right] \mathbf{P}
$$

Based on these matrices the stationary fluid density vector and the stationary probability vector of the idle buffer can be computed by the following theorem.

Theorem 1. If the drift of the queue is negative, vector $\pi(x)$ is given by

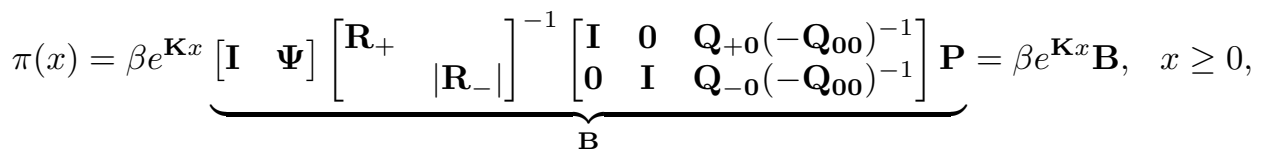

and the probability mass vector $p$ equals to

$$
p=\left[\begin{array}{lll}
0 & p_{-} & p_{0}
\end{array}\right] \mathbf{P}
$$

\footnotetext{
${ }^{3}$ It would be straight forward to assume that matrices $\mathbf{Q}$ and $\mathbf{R}$ are given in the partitioned form apriori, as it is often assumed in the literature of fluid models. However, later in the paper we exploit the Kronecker structure of these matrices, which is destroyed by the partitioning. We therefore introduce the permutation matrix $\mathbf{P}$ to go back and forth between the partitioned and the Kronecker structure of $\mathbf{Q}$ and $\mathbf{R}$.
} 
where $\beta, p_{-}$and $p_{0}$ are the solutions to the set of linear equations

$$
\begin{aligned}
& {\left.\left[\begin{array}{lll}
\beta & p_{-} & p_{0}
\end{array}\right]\left[\begin{array}{ccc}
-\mathbf{B R} \\
\mathbf{Q}_{-+}^{(\mathbf{0})} & \mathbf{Q}_{-}^{(\mathbf{0})} & \mathbf{Q}_{-\mathbf{0}}^{(\mathbf{0})} \\
\mathbf{Q}_{\mathbf{0}+}^{(\mathbf{0})} & \mathbf{Q}_{\mathbf{0}-}^{(\mathbf{0})} & \mathbf{Q}_{\mathbf{0 0}}^{(\mathbf{0})}
\end{array}\right]\right] }=0 \\
& \beta(-\mathbf{K})^{-1} \mathbf{B} \mathbb{1}+p_{-} \mathbb{1}+p_{0} \mathbb{1}=1 .
\end{aligned}
$$

Proof. Eq. (11) comes from [10], while (13) is obtained by substituting (11) into (3) and (4). Eq. (14) follows from (5).

An other useful relation which is used later in this paper is obtained when we substitute the solution (11) back to the differential equation (2), yielding $\beta e^{\mathbf{K} x} \mathbf{K B R}=\beta e^{\mathbf{K} x} \mathbf{B Q}$ for $\forall x \geq 0$, from which we have

$$
\mathbf{K B R}=\mathbf{B Q},
$$

since $\beta \neq 0$ and $\mathbf{K}$ has full rank. Finally, by substituting the solution (11) back to the boundary equation (3) gives

$$
\beta \mathbf{B R}=p \mathbf{Q}
$$

which will be frequently used below.

\section{Sojourn Time Distribution With Dependent Input and Output}

The sojourn time of a fluid drop in this system assuming FCFS principle is the time measured from the arrival of the fluid drop to its departure from the buffer. The sojourn time distribution of QBD queues is known to be matrix-exponential of order $N^{2}$ [4]. In [4] it is also proven that the sojourn time distribution has a phase-type representation if some conditions hold. In this section we perform similar analysis steps as in [4] for continuous queues in order to derive similar results for the sojourn time distribution of MFQs.

\subsection{The Matrix-Exponentially Distributed Sojourn Time}

To derive the sojourn time distribution we have to calculate how long it takes to serve the amount of fluid an arriving fluid drop finds in the queue.

The stationary density of the fluid level at fluid drop arrival instants $\hat{\pi}(x)$ can be derived from $\pi(x)$ as

$$
\hat{\pi}(x)=\frac{1}{\lambda} \pi(x) \mathbf{R}_{\text {in }}=\underbrace{\frac{1}{\lambda} \beta}_{\hat{\beta}} e^{\mathbf{K} x} \underbrace{\mathbf{B R}_{\text {in }}}_{\hat{\mathbf{B}}}=\hat{\beta} e^{\mathbf{K} x} \hat{\mathbf{B}}, \quad x \geq 0,
$$

where $\lambda$ is the normalizing constant given by

$$
\lambda=p \mathbf{R}_{\mathbf{i n}} \mathbb{1}+\beta(-\mathbf{K})^{-1} \hat{\mathbf{B}} \mathbb{1},
$$

which is in fact the mean arrival rate, and can alternatively be obtained by $\lambda=\xi \mathbf{R}_{\mathbf{i n}} \mathbb{1}$.

Next, we have to calculate how long it takes to serve a given amount of fluid from the buffer starting from an initial state. The service process of the queue $\{\mathcal{Y}(t), t \geq 0\}$ is the amount of fluid that can leave the queue up to time $t$ given that there is always enough fluid in the queue. The service process is characterized by $\left(\mathbf{Q}, \mathbf{R}_{\text {out }}\right)$. Let us introduce the following probability:

$$
N_{i, j}(x, t)=P(\mathcal{Y}(t)<x, \mathcal{Z}(t)=j \mid \mathcal{Z}(0)=i),
$$

and the corresponding matrix $\mathbf{N}(x, t)=\left\{N_{i, j}(x, t), i, j \in \mathcal{S}\right\}$. 
Observe that matrix $\mathbf{R}_{\text {out }}$ has only non-negative entries, thus $\left(\mathbf{Q}, \mathbf{R}_{\text {out }}\right)$ defines a Markov reward model. Using the terminology of the Markov reward models $N_{i, j}(x, t)$ is the distribution of the accumulated reward, that is known to satisfy the following system of differential equations [11]:

$$
\frac{\partial}{\partial t} \mathbf{N}(x, t)+\frac{\partial}{\partial x} \mathbf{N}(x, t) \mathbf{R}_{\text {out }}=\mathbf{N}(x, t) \mathbf{Q} .
$$

The next theorem provides the distribution of the sojourn time.

Theorem 2. The distribution of the sojourn time of a fluid drop is given by

$$
P(\mathcal{V} \leq t)=1-\left(\mathbb{1}^{T} \otimes \hat{\beta}(-\mathbf{K})^{-1}\right) e^{\left(\mathbf{R}_{\text {out }} \otimes \mathbf{K}+\mathbf{Q}^{T} \otimes \mathbf{I}\right) t} v e c\langle\hat{\mathbf{B}}\rangle,
$$

where vec $\langle$ is the column stacking operator.

Proof. The sojourn time of a fluid drop is less than or equal to $t$ if the service process is able to serve at least as much fluid as the arrival fluid drop found in the queue in time $t$. Thus, by conditioning on the fluid level at fluid drop arrivals the distribution of the waiting time is given by

$$
P(\mathcal{V} \leq t)=1-\int_{0}^{\infty} \hat{\pi}(x) \mathbf{N}(x, t) \mathbb{1} d x .
$$

Substituting (17) into (22) yields

$$
P(\mathcal{V} \leq t)=1-\hat{\beta} \underbrace{\int_{0}^{\infty} e^{\mathbf{K} x} \hat{\mathbf{B}} \mathbf{N}(x, t) d x}_{\mathbf{W}(t)} \mathbb{1}=1-\hat{\beta} \mathbf{W}(t) \mathbb{1}
$$

where

$$
\mathbf{W}(t)=\int_{0}^{\infty} e^{\mathbf{K} x} \hat{\mathbf{B}} \mathbf{N}(x, t) d x
$$

Multiplying (20) by $e^{\mathbf{K} x} \hat{\mathbf{B}}$ from the left and taking the integral from $x=0$ to $\infty$ leads to a system of differential equations for $\mathbf{W}(t)$

$$
\underbrace{\int_{0}^{\infty} e^{\mathbf{K} x} \hat{\mathbf{B}} \frac{\partial}{\partial t} \mathbf{N}(x, t) d x}_{\frac{d}{d t} \mathbf{W}(t)}+\underbrace{\int_{0}^{\infty} e^{\mathbf{K} x} \hat{\mathbf{B}} \frac{\partial}{\partial x} \mathbf{N}(x, t) d x}_{-\mathbf{K} \mathbf{W}(t)} \mathbf{R}_{\text {out }}=\underbrace{\int_{0}^{\infty} e^{\mathbf{K} x} \hat{\mathbf{B}} \mathbf{N}(x, t) \mathbf{Q} d x}_{\mathbf{W}(t) \mathbf{Q}},
$$

that is

$$
\frac{d}{d t} \mathbf{W}(t)-\mathbf{K W}(t) \mathbf{R}_{\mathbf{o u t}}=\mathbf{W}(t) \mathbf{Q}
$$

The second term at the left hand side of (25) has been integrated by parts, exploiting that $\left[e^{\mathbf{K} x} \hat{\mathbf{B}} \mathbf{N}(x, t)\right]_{0}^{\infty}=$ 0.

Making use of the properties of the vec \langle\rangle operator [12] leads to a closed-form solution for (26)

$$
\frac{d}{d t} \operatorname{vec}\langle\mathbf{W}(t)\rangle=\left(\mathbf{R}_{\text {out }} \otimes \mathbf{K}+\mathbf{Q}^{T} \otimes \mathbf{I}\right) \operatorname{vec}\langle\mathbf{W}(t)\rangle,
$$

from which $\operatorname{vec}\langle\mathbf{W}(t)\rangle$ is

$$
\operatorname{vec}\langle\mathbf{W}(t)\rangle=e^{\left(\mathbf{R}_{\text {out }} \otimes \mathbf{K}+\mathbf{Q}^{T} \otimes \mathbf{I}\right) t} \operatorname{vec}\langle\mathbf{W}(0)\rangle .
$$


Since $\mathbf{W}(0)=\int_{0}^{\infty} e^{\mathbf{K} x} \hat{\mathbf{B}} d x=(-\mathbf{K})^{-1} \hat{\mathbf{B}}$ and since (23) can be expressed by the vec \langle\rangle operator as $P(\mathcal{V}>t)=\left(\mathbb{1}^{T} \otimes \hat{\beta}\right) \operatorname{vec}\langle\mathbf{W}(t)\rangle$ we obtain

$$
P(\mathcal{V} \leq t)=1-\left(\mathbb{1}^{T} \otimes \hat{\beta}\right) e^{\left(\mathbf{R}_{\text {out }} \otimes \mathbf{K}+\mathbf{Q}^{T} \otimes \mathbf{I}\right) t} \operatorname{vec}\left\langle(-\mathbf{K})^{-1} \hat{\mathbf{B}}\right\rangle .
$$

Finally, applying vec $\left\langle(-\mathbf{K})^{-1} \hat{\mathbf{B}}\right\rangle=\left(\mathbf{I} \otimes(-\mathbf{K})^{-1}\right) \operatorname{vec}\langle\hat{\mathbf{B}}\rangle$ and recognizing that $\mathbf{I} \otimes(-\mathbf{K})^{-1}$ and the exponential term commute we can establish (21).

Note that

$$
P(\mathcal{V} \leq 0)=1-\hat{\beta} \mathbf{W}(0) \mathbb{1}=1-\hat{\beta}(-\mathbf{K})^{-1} \hat{\mathbf{B}} \mathbb{1}=1-\int_{0}^{\infty} \hat{\pi}(x) \mathbb{1} d x,
$$

which means that having an exactly 0 sojourn time has a positive probability (which equals to the probability of empty buffer at arrival instance).

\subsection{Phase-Type Representation}

The sojourn time distribution given by (21) has a phase-type (PH) representation.

Theorem 3. The sojourn time of a fluid drop is phase-type distributed with

$$
P(\mathcal{V} \leq t)=1-z e^{\mathbf{z} t} \mathbb{1}
$$

where

$$
\begin{aligned}
z & =\operatorname{vec}\langle\hat{\mathbf{B}}\rangle^{T}(\mathbf{I} \otimes \boldsymbol{\Delta}), \\
\mathbf{Z} & =\mathbf{R}_{\text {out }} \otimes\left(\boldsymbol{\Delta}^{-1} \mathbf{K}^{T} \boldsymbol{\Delta}\right)+\mathbf{Q} \otimes \mathbf{I},
\end{aligned}
$$

and $\boldsymbol{\Delta}=\operatorname{diag}\left\langle\hat{\beta}(-\mathbf{K})^{-1}\right\rangle$.

Proof. Transposing the second term of (21) results the following expression after some algebraic manipulation.

$$
\begin{aligned}
& P(\mathcal{V}>t)=\left(\mathbb{1}^{T} \otimes \hat{\beta}(-\mathbf{K})^{-1}\right) e^{\left(\mathbf{R}_{\text {out }} \otimes \mathbf{K}+\mathbf{Q}^{T} \otimes \mathbf{I}\right) t} \operatorname{vec}\langle\hat{\mathbf{B}}\rangle \\
& =\operatorname{vec}\langle\hat{\mathbf{B}}\rangle^{T} e^{\left(\mathbf{R}_{\text {out }} \otimes \mathbf{K}^{T}+\mathbf{Q} \otimes \mathbf{I}\right) t}\left(\mathbb{1} \otimes(-\mathbf{K})^{-1^{T}} \hat{\beta}^{T}\right) \\
& =\underbrace{\operatorname{vec}\langle\hat{\mathbf{B}}\rangle^{T}(\mathbf{I} \otimes \boldsymbol{\Delta})}_{z} \underbrace{e^{\left(\mathbf{R}_{\text {out }} \otimes\left(\boldsymbol{\Delta}^{-1} \mathbf{K}^{T} \boldsymbol{\Delta}\right)+\mathbf{Q} \otimes \mathbf{I}\right) t}}_{e^{\mathbf{Z t}}} \underbrace{\left(\mathbf{I} \otimes \boldsymbol{\Delta}^{-1}\right)\left(\mathbb{1} \otimes(-\mathbf{K})^{-1^{T}} \hat{\beta}^{T}\right)}_{\mathbb{1}}=z e^{\mathbf{Z} t} \mathbb{1} .
\end{aligned}
$$

$z$ is a proper initial probability vector for the $\mathrm{PH}$ distribution because the entries of $\hat{\mathbf{B}}$ and $\boldsymbol{\Delta}$ are nonnegative, $z$ is non-negative as well. Furthermore, using the identities of the vec \langle\rangle operator gives

$$
\begin{aligned}
z \mathbb{1} & =\operatorname{vec}\langle\hat{\mathbf{B}}\rangle^{T}\left(\mathbf{I} \otimes \operatorname{diag}\left\langle\hat{\beta}(-\mathbf{K})^{-1}\right\rangle\right) \mathbb{1}=\mathbb{1}^{T}\left(\mathbf{I} \otimes \operatorname{diag}\left\langle\hat{\beta}(-\mathbf{K})^{-1}\right\rangle\right) \operatorname{vec}\langle\hat{\mathbf{B}}\rangle \\
& =\left(\mathbb{1}^{T} \otimes \hat{\beta}(-\mathbf{K})^{-1}\right) \operatorname{vec}\langle\hat{\mathbf{B}}\rangle=\operatorname{vec}\left\langle\hat{\beta}(-\mathbf{K})^{-1} \hat{\mathbf{B}} \mathbb{1}\right\rangle=\int_{x=0}^{\infty} \hat{\pi}(x) \mathbb{1} d x \leq 1 .
\end{aligned}
$$

It remains to show that matrix $\mathbf{Z}$ is a valid sub-generator of a continuous time Markov chain. Since the off diagonal entries of $\mathbf{Q}$ and $\mathbf{K}$ are non-negative (see (9)), so are the off diagonal entries of $\mathbf{Z}$. For the row-sums of $\mathbf{Z}$ we have

$$
\mathbf{Z} \mathbb{1}=\mathbf{R}_{\text {out }} \mathbb{1} \otimes\left(\boldsymbol{\Delta}^{-1} \mathbf{K}^{T} \boldsymbol{\Delta} \mathbb{1}\right)+\mathbf{Q} \mathbb{1} \otimes \mathbb{1}=\mathbf{R}_{\text {out }} \mathbb{1} \otimes\left(\boldsymbol{\Delta}^{-1} \mathbf{K}^{T} \boldsymbol{\Delta} \mathbb{1}\right),
$$


since $\mathbf{Q} \mathbb{1}=0$, meaning that $\mathbf{Z} \mathbb{1} \leq 0$ holds if and only if $\boldsymbol{\Delta}^{-1} \mathbf{K}^{T} \boldsymbol{\Delta} \mathbb{1} \leq 0$. Substituting the definition of $\boldsymbol{\Delta}$ yields

$$
\begin{aligned}
\boldsymbol{\Delta}^{-1} \mathbf{K}^{T} \boldsymbol{\Delta} \mathbb{1} & =\operatorname{diag}\left\langle\hat{\beta}(-\mathbf{K})^{-1}\right\rangle^{-1} \mathbf{K}^{T} \operatorname{diag}\left\langle\hat{\beta}(-\mathbf{K})^{-1}\right\rangle \mathbb{1} \\
& =\operatorname{diag}\left\langle\hat{\beta}(-\mathbf{K})^{-1}\right\rangle^{-1} \mathbf{K}^{T}(-\mathbf{K})^{-1^{T}} \hat{\beta}^{T} \\
& =-\operatorname{diag}\left\langle\hat{\beta}(-\mathbf{K})^{-1}\right\rangle^{-1} \hat{\beta}^{T}
\end{aligned}
$$

which is non-positive since $\hat{\beta}$ and $(-\mathbf{K})^{-1}$ are non-negative.

The conclusion of this section is that the sojourn time of an MFQ is PH distributed and it has a size $N \cdot N_{+}$ representation.

\section{MFQs with Independent Arrival and Service Processes}

In this section we consider the case when the input and the output processes are controlled by two independent irreducible CTMCs, $\left\{\mathcal{Z}^{(i n)}(t) \in \mathcal{S}^{(i n)}, t>0,\left|\mathcal{S}^{(i n)}\right|=N_{\text {in }}\right\}$ and $\left\{\mathcal{Z}^{(\text {out })}(t) \in \mathcal{S}^{(\text {out })}, t>\right.$ $\left.0,\left|\mathcal{S}^{(\text {out })}\right|=N_{\text {out }}\right\}$ with the corresponding generators denoted by $\mathbf{Q}^{(\text {in })}$ and $\mathbf{Q}^{(\text {out })}$, respectively. The input and output fluid rates associated with the states of the background processes are held by diagonal matrices $\mathbf{R}^{(\text {in) }}=\operatorname{diag}\left\langle r_{i}^{(i n)}\right\rangle, i \in \mathcal{S}_{\text {in }}$ and $\mathbf{R}^{(\text {out })}=\operatorname{diag}\left\langle r_{i}^{(\text {out })}\right\rangle, i=\in \mathcal{S}_{\text {out }}$. Consequently, in case of independent fluid input and output processes

$$
\begin{aligned}
& \mathbf{R}=\mathbf{R}^{(\mathbf{i n})} \otimes \mathbf{I}-\mathbf{I} \otimes \mathbf{R}^{(\text {out })}, \\
& \mathbf{Q}=\mathbf{Q}^{(\text {in })} \otimes \mathbf{I}+\mathbf{I} \otimes \mathbf{Q}^{(\text {out })}
\end{aligned}
$$

The mean input and output fluid rates are obtained by $\lambda=\xi^{(\text {in })} \mathbf{R}^{(\text {in })} \mathbb{1}$ and $\mu=\xi^{(\text {out })} \mathbf{R}^{(\text {out })} \mathbb{1}$, where $\xi^{(\text {in })}$ and $\xi^{(\text {out })}$ are the steady state distributions of $\mathcal{Z}^{(\text {in })}$ and $\mathcal{Z}^{(\text {out })}$, respectively, that is $\xi^{(\text {in })} \mathbf{Q}^{(\text {in })}=0$, $\xi^{(\text {in) }} \mathbb{1}=1, \xi^{(\text {out })} \mathbf{Q}^{(\text {out })}=0$ and $\xi^{(\text {out })} \mathbb{1}=1 . \lambda<\mu$ is assumed throughout the paper.

We consider two different output process behaviors if the buffer is idle (i.e., there is no fluid in the buffer and the input process generates less fluid than the output process can serve).

- Boundary-continuous case. In this case the evolution of the background CTMC of the output process is independent of the fluid level and it always follows the differential equation

$$
\frac{d}{d t} \xi_{i}^{(\text {out })}(t)=\sum_{j \in \mathcal{S}^{(\text {out })}} \xi_{j}^{(\text {out })}(t) \mathbf{Q}_{j i}^{(\text {out })}, \quad \forall i \in \mathcal{S}^{(\text {out })}
$$

where $\xi_{i}^{(\text {out })}(t)=P\left(\mathcal{Z}^{(\text {out })}(t)=i\right)$. In this case the MFQ representing the fluid buffer is characterized by

$$
\mathbf{R}_{\text {cont }}=\mathbf{R}, \mathbf{Q}_{\text {cont }}=\mathbf{Q}, \mathbf{Q}_{\text {cont }}^{(\mathbf{0})}=\mathbf{Q}_{\text {cont }},
$$

and the output process can be considered as a random environment modulating the (possibly unutilized) output capabilities of the buffer.

- Boundary-proportional case. In this case the background CTMC of the output process depends on the amount of fluid actually served from the buffer. If the amount of available fluid is less than the amount the output process is able to serve (that is $\mathcal{X}(t)=0$ and $r_{\mathcal{Z}^{(o u t)}(t)}^{(\text {out }}>r_{\mathcal{Z}^{(i n)}(t)}^{(i n)}$ ), then the background process proportionally slows down, otherwise it follows eq. (40). That is for $\forall i \in \mathcal{S}^{(\text {out })}$

$$
\frac{d}{d t} \xi_{i}^{(\text {out })}(t)= \begin{cases}\sum_{j \in \mathcal{S}^{(\text {out })}} \xi_{j}^{(\text {out })}(t) \mathbf{Q}_{j i}^{(\text {out })}, & \text { if } \mathcal{X}(t)>0, \\ \sum_{j \in \mathcal{S}^{(\text {out })}} \xi_{j}^{(\text {out })}(t) \mathbf{Q}_{j i}^{(\text {out })} \max \left\{1, \frac{r_{\mathcal{Z}^{(i n)}(t)}^{(\text {in })}}{\left.r_{j}^{(\text {out })}\right\},}\right. & \text { if } \mathcal{X}(t)=0 .\end{cases}
$$


In this case the MFQ corresponding to the buffer is characterized by

$$
\mathbf{R}_{\text {prop }}=\mathbf{R}, \mathbf{Q}_{\text {prop }}=\mathbf{Q}, \mathbf{Q}_{\text {prop }}^{(\mathbf{0})}=\mathbf{Q}^{(\text {in })} \otimes \mathbf{I}+\mathbf{R}^{(\text {in })} \otimes \mathbf{R}^{(\text {out })^{-1}} \mathbf{Q}^{(\text {out })},
$$

where the generator matrix is irregular at the boundary. Note that this is not an artificial system: it is the continuous counterpart of the MAP/MAP/1 queue, where the MAP controlling the service process freezes when the queue becomes idle.

It is important to note that according to (7)-(10) matrices $\mathbf{R}$ and $\mathbf{Q}$ completely determine matrices $\boldsymbol{\Psi}$, $\mathbf{K}, \mathbf{B}$ and only vectors $p$ and $\beta$ depend on $\mathbf{Q}^{(\mathbf{0})}$ via (13).

While the sojourn time distribution can be obtained by applying the results of Section 3 under both boundary behaviors, in the following subsections we are going to show that the independence of the input and output processes can be exploited to arrive to a much smaller representation for the sojourn time distribution.

\subsection{A Fluid Model with Fundamental Role in Sojourn Time Analysis}

In this section we define and investigate a special Markov fluid model. The significance of this fluid model is that its stationary fluid level distribution is closely related with the sojourn time distributions of MFQs with independent input and output arrival processes.

This special fluid model is defined by generator matrix ${ }^{4} \tilde{\mathbf{Q}}$ and fluid rate matrix $\tilde{\mathbf{R}}$

$$
\begin{aligned}
\tilde{\mathbf{R}} & =\mathbf{R}^{(\text {in })} \otimes \mathbf{I}-\mathbf{I} \otimes \mathbf{R}^{(\text {out })} \\
\tilde{\mathbf{Q}} & =\mathbf{Q}^{(\text {in })} \otimes \mathbf{R}^{(\text {out })}+\mathbf{R}^{(\text {in })} \otimes \mathbf{Q}^{(\text {out })}
\end{aligned}
$$

Note that $\tilde{\mathbf{R}}=\mathbf{R}$, thus we can use a single fluid rate matrix $\mathbf{R}$ in the sequel.

To relate the stationary fluid level distribution of the fluid model defined by $(\mathbf{R}, \mathbf{Q})$ and the one of the special fluid model defined by $(\mathbf{R}, \tilde{\mathbf{Q}})$ we need the relations of matrices $\boldsymbol{\Psi}, \mathbf{K}, \mathbf{B}$ and matrices $\tilde{\boldsymbol{\Psi}}, \tilde{\mathbf{K}}, \tilde{\mathbf{B}}$, which are computed from $\tilde{\mathbf{Q}}, \mathbf{R}$ via (7)-(10).

The next theorem presents surprising results which turn out to be fundamental in the rest of the paper.

Theorem 4. The following relations hold among matrices $\boldsymbol{\Psi}, \mathbf{K}, \mathbf{B}$ and matrices $\tilde{\boldsymbol{\Psi}}, \tilde{\mathbf{K}}, \tilde{\mathbf{B}}$

$$
\begin{aligned}
\tilde{\mathbf{\Psi}} & =\mathbf{\Psi}, \\
\left(\mathbf{R}^{\bullet-1} \mathbf{Q}^{\bullet}\right)\left(\mathbf{R}^{\bullet-1} \tilde{\mathbf{Q}}\right)^{\bullet} & =\left(\mathbf{R}^{\bullet-1} \tilde{\mathbf{Q}}^{\bullet}\right)\left(\mathbf{R}^{\bullet-1} \mathbf{Q}^{\bullet}\right), \\
\mathbf{K} \tilde{\mathbf{K}} & =\tilde{\mathbf{K}} \mathbf{K} . \\
\tilde{\mathbf{B}} & =\mathbf{B},
\end{aligned}
$$

The proof of the theorem is provided in Appendix Appendix A.

\subsection{Sojourn time distribution with Boundary-proportional output process}

In case of queues with discrete jobs an elegant and efficient technique to evaluate the sojourn time distribution is based on the age process [5]. The age process $\{\mathcal{A}(t), t>0\}$ represents the age of the job under service, which, at job departure instant equals to the time the job spent in the system.

We follow the same approach for fluid queues in this section, however, the jobs are infinitesimally small fluid drops in our case. Masuyama and Takine call the same process as "attained waiting time" in [13], however the analysis applied there requires a constant (state independent) output fluid rate. We solve the

\footnotetext{
${ }^{4}$ If both $\mathbf{R}^{(\mathbf{i n})}$ and $\mathbf{R}^{\text {(out) }}$ contains at least one state with zero rate then $\tilde{\mathbf{Q}}$ is reducible. In this case $\tilde{\mathbf{Q}}$ contains an associated zero column and row, i.e., unreachable absorbing state. This technical issue might be handled in different ways. Eliminating the redundant states is reasonable, but destroys the Kronecker representation. Our particular implementation carries on the Kronecker representation and our fluid solver is prepared for the potential presence of redundant states.
} 
general case in this paper, and show that the age process is related to the fluid level process of the special fluid model characterized by matrices $\mathbf{R}$ and $\tilde{\mathbf{Q}}$.

The next theorem provides the probability density function of the fluid drop sojourn time in the buffer

$$
v_{\text {prop }}(x)=\lim _{\Delta \rightarrow 0} \frac{1}{\Delta} P\left(\mathcal{V}_{\text {prop }} \in(x, x+\Delta)\right)
$$

based on the stationary fluid density vector of the fluid model characterized by $\left(\mathbf{R}, \tilde{\mathbf{Q}}, \tilde{\mathbf{Q}}{ }^{(\mathbf{0})}=\tilde{\mathbf{Q}}\right)$, which has the form of

$$
\tilde{\pi}(x)=\tilde{\beta} e^{\tilde{\mathbf{K}} x} \underbrace{\left[\begin{array}{cc}
\mathbf{I} & \tilde{\mathbf{\Psi}}
\end{array}\right]\left[\begin{array}{cc}
\mathbf{R}_{+} & \\
0 & \left|\mathbf{R}_{-}\right|
\end{array}\right]^{-1}\left[\begin{array}{ccc}
\mathbf{I} & \mathbf{0} & \tilde{\mathbf{Q}}_{+\mathbf{0}}\left(-\tilde{\mathbf{Q}}_{\mathbf{0 0}}\right)^{-1} \\
\mathbf{0} & \mathbf{I} & \tilde{\mathbf{Q}}_{-\mathbf{0}}\left(-\tilde{\mathbf{Q}}_{00}\right)^{-1}
\end{array}\right]}_{\tilde{\mathbf{B}}} \mathbf{P}=\tilde{\beta} e^{\tilde{\mathbf{K}} x} \tilde{\mathbf{B}}, \quad x \geq 0,
$$

according to (11), where $\tilde{\beta}, \tilde{\mathbf{K}}$ and $\tilde{\mathbf{B}}$ are computed from $\left(\mathbf{R}, \tilde{\mathbf{Q}}, \tilde{\mathbf{Q}}^{(\mathbf{0})}=\tilde{\mathbf{Q}}\right)$ by $(7)-(13)$. In $(51), \tilde{\mathbf{\Psi}}=\mathbf{\Psi}$ and $\tilde{\mathbf{B}}=\mathbf{B}$ due to Theorem 4 .

Theorem 5. The stationary probability density of the sojourn time in the fluid queue with independent input and output processes and boundary-proportional output policy (defined by $\left(\mathbf{R}, \mathbf{Q}, \mathbf{Q}_{\mathbf{p r o p}}^{(\mathbf{0})}\right.$ ) is given by

$$
v_{\text {prop }}(x)=\frac{1}{\lambda \mu} \tilde{\pi}(x)\left(\mathbf{R}^{(\mathbf{i n})} \otimes \mathbf{R}^{(\text {out })}\right) \mathbb{1}=\frac{1}{\lambda \mu} \tilde{\beta} e^{\tilde{\mathbf{K}} x} \mathbf{B}\left(\mathbf{R}^{(\mathbf{i n})} \otimes \mathbf{R}^{(\text {out })}\right) \mathbb{1},
$$

where $\tilde{\pi}(x)$ is the stationary fluid density vector of the fluid model defined by the fluid model $\left(\mathbf{R}, \tilde{\mathbf{Q}}, \tilde{\mathbf{Q}}{ }^{(\mathbf{0})}=\tilde{\mathbf{Q}}\right)$ in (51).

The proof is provided in Appendix Appendix B. The sojourn time distribution has a phase-type representation also in this case.

Theorem 6. The sojourn time of a fluid drop is phase-type distributed with representation

$$
P\left(\mathcal{V}_{\text {prop }} \leq x\right)=1-z_{\text {prop }} e^{\mathbf{Z}_{\text {prop }} \cdot x} \mathbb{1}
$$

where

$$
\begin{aligned}
z_{\text {prop }} & =\mathbb{1}^{T}\left(\mathbf{R}^{(\text {in })^{T}} \otimes \mathbf{R}^{(\text {out })^{T}}\right) \frac{1}{\lambda \mu} \mathbf{B}^{T} \boldsymbol{\Delta}, \\
\mathbf{Z}_{\text {prop }} & =\boldsymbol{\Delta}^{-1} \tilde{\mathbf{K}}^{T} \boldsymbol{\Delta},
\end{aligned}
$$

and $\boldsymbol{\Delta}=\operatorname{diag}\left\langle\tilde{\beta}(-\tilde{\mathbf{K}})^{-1}\right\rangle$.

PROOF. We start by transposing the complementary cdf of the sojourn time distribution based on (52)

$$
\begin{aligned}
& P\left(\mathcal{V}_{\text {prop }}>x\right)=\mathbb{1}^{T} \frac{1}{\lambda \mu}\left(\mathbf{R}^{(\text {in })^{T}} \otimes \mathbf{R}^{(\text {out })^{T}}\right) \mathbf{B}^{T} e^{\tilde{\mathbf{K}}^{T} x}(-\tilde{\mathbf{K}})^{-1^{T}} \tilde{\beta}^{T} \\
& =\underbrace{\mathbb{1}^{T} \frac{1}{\lambda \mu}\left(\mathbf{R}^{(\text {in })^{T}} \otimes \mathbf{R}^{(\text {out })^{T}}\right) \mathbf{B}^{T} \boldsymbol{\Delta}}_{z_{\text {prop }}} \underbrace{e^{\boldsymbol{\Delta}^{-1} \tilde{\mathbf{K}}^{T} \boldsymbol{\Delta} x}}_{e^{\mathbf{z}_{\text {prop } x}}} \underbrace{\boldsymbol{\Delta}^{-1}(-\tilde{\mathbf{K}})^{-1^{T}} \tilde{\beta}^{T}}_{\mathbb{1}} .
\end{aligned}
$$

Vector $z_{\text {prop }}$ is non-negative, since all matrices in its definition are non-negative. To show that it is a valid initial probability vector we have to prove that $z_{\text {prop }} \mathbb{1} \leq 1$. Transposing $z_{\text {prop }} \mathbb{1}$ gives

$$
z_{\text {prop }} \mathbb{1}=\mathbb{1}^{T} \frac{1}{\lambda \mu} \operatorname{diag}\left\langle\tilde{\beta}(-\tilde{\mathbf{K}})^{-1}\right\rangle \mathbf{B}\left(\mathbf{R}^{(\text {in })} \otimes \mathbf{R}^{(\text {out })}\right) \mathbb{1}=\int_{x=0}^{\infty} v_{\text {prop }}(x) \mathbb{1} d x \leq 1 .
$$


Next we show that matrix $\mathbf{Z}_{\text {prop }}$ is a valid sub-generator of a continuous time Markov chain. Since the off diagonal entries of $\tilde{\mathbf{K}}$ are non-negative and $\boldsymbol{\Delta}$ is a non-negative diagonal matrix, the off-diagonal entries of $\mathbf{Z}_{\text {prop }}$ are non-negative as well. The row-sums of $\mathbf{Z}_{\text {prop }}$ are

$$
\boldsymbol{\Delta}^{-1} \tilde{\mathbf{K}}^{T} \boldsymbol{\Delta} \mathbb{1}=\boldsymbol{\Delta}^{-1} \tilde{\mathbf{K}}^{T} \operatorname{diag}\left\langle\tilde{\beta}(-\tilde{\mathbf{K}})^{-1}\right\rangle \mathbb{1}=\boldsymbol{\Delta}^{-1} \tilde{\mathbf{K}}^{T}(-\tilde{\mathbf{K}})^{-1^{T}} \tilde{\beta}^{T}=-\boldsymbol{\Delta}^{-1} \tilde{\beta}^{T}
$$

which is non-positive since $\hat{\beta}$ and $\boldsymbol{\Delta}^{-1}$ are non-negative.

As mentioned earlier, the sojourn time distribution can be computed by the results of Section 3 as well, however it gives a size $N \cdot N_{+} \mathrm{PH}$ representation. In case of independent input and output process Theorem 6 provides a size $N_{+} \mathrm{PH}$ representation, which is a significant size reduction.

\subsection{Sojourn time distribution with Boundary-continuous output process}

In the boundary-continuous case the background CTMC of the output process does not slow down when the buffer is empty, but evolves according to the same generator matrix. We define the density function of the sojourn time in the boundary-continuous case as

$$
v_{\text {cont }}(x)=\lim _{\Delta \rightarrow 0} \frac{1}{\Delta} P\left(\mathcal{V}_{\text {cont }} \in(x, x+\Delta)\right) .
$$

For the analysis of this case, similar to Section 4.2, we introduce a special fluid model, whose fluid density vector is closely related with the sojourn time distribution in the boundary-continuous queue. In contrast with Section 4.2, where the original model had an irregular behavior at zero and the special model had a regular one, in the boundary-continuous case the original model is regular and the special model is irregular at level zero. The special fluid model is defined by $\left(\mathbf{R}, \tilde{\mathbf{Q}}, \tilde{\mathbf{Q}}_{\mathbf{i r}}^{(\mathbf{0})}\right)$ where

$$
\tilde{\mathbf{Q}}_{\mathrm{ir}}^{(\mathbf{0})}=\mathbf{Q}^{(\mathrm{in})} \otimes \mathbf{R}^{(\text {out })}+\mathbf{I} \otimes \mathbf{R}^{(\text {out })} \mathbf{Q}^{(\text {out })}
$$

The stationary fluid density vector of this special fluid model is

$$
\tilde{\pi}_{i r}(x)=\tilde{\beta}_{i r} e^{\tilde{\mathbf{K}} x} \underbrace{\left[\begin{array}{ll}
\mathbf{I} & \tilde{\mathbf{\Psi}}
\end{array}\right]\left[\begin{array}{cc}
\mathbf{R}_{+} & \\
0 & \left|\mathbf{R}_{-}\right|
\end{array}\right]^{-1}\left[\begin{array}{ccc}
\mathbf{I} & \mathbf{0} & \tilde{\mathbf{Q}}_{+\mathbf{0}}\left(-\tilde{\mathbf{Q}}_{00}\right)^{-1} \\
\mathbf{0} & \mathbf{I} & \tilde{\mathbf{Q}}_{-\mathbf{0}}\left(-\tilde{\mathbf{Q}}_{\mathbf{0 0}}\right)^{-1}
\end{array}\right]}_{\tilde{\mathbf{B}}}, \quad x \geq 0,
$$

where $\tilde{\beta}_{i r}$, $\tilde{\mathbf{K}}$ and $\tilde{\mathbf{B}}$ and the corresponding probability mass vector at zero, $\tilde{p}_{i r}$, are computed from $\left(\mathbf{R}, \tilde{\mathbf{Q}}, \tilde{\mathbf{Q}}_{\mathbf{i r}}^{(\mathbf{0})}\right)$ by (7)-(13) and $\tilde{\boldsymbol{\Psi}}=\boldsymbol{\Psi}$ and $\tilde{\mathbf{B}}=\mathbf{B}$ due to Theorem 4. Note that the only difference compared to $(51)$ is the initial vector since all other components of the solution are boundary behavior independent.

Theorem 7. The stationary probability density function of the sojourn time of a fluid queue with independent input and output processes and boundary-continuous output policy is given by

$$
v_{\text {cont }}(x)=\frac{1}{c_{\text {ir }}} \tilde{\pi}_{i r}(x)\left(\mathbf{R}^{(\text {in })} \otimes \mathbf{R}^{(\text {out })}\right) \mathbb{1}=\frac{1}{c_{\text {ir }}} \tilde{\beta}_{i r} e^{\tilde{\mathbf{K}} x} \mathbf{B}\left(\mathbf{R}^{(\mathbf{i n})} \otimes \mathbf{R}^{(\text {out })}\right) \mathbb{1},
$$

where $\tilde{\pi}_{i r}(x)$ is the stationary density of the fluid level of the fluid model defined by $\mathbf{R}, \tilde{\mathbf{Q}}$ and $\tilde{\mathbf{Q}}_{\mathbf{i r}}^{(\mathbf{0})}$, and $c_{\text {ir }}$ denotes the normalizing constant

$$
c_{i r}=\tilde{p}_{i r}\left(\mathbf{R}^{(\text {in })} \otimes \mathbf{R}^{(\text {out })}\right) \mathbb{1}+\tilde{\beta}_{i r}(-\tilde{\mathbf{K}})^{-1} \mathbf{B}\left(\mathbf{R}^{(\text {in })} \otimes \mathbf{R}^{(\text {out })}\right) \mathbb{1} .
$$

The proof is provided in Appendix Appendix C.

At this point, by using the results of Theorem 7, we can express the density of the sojourn time distribution by solving a fluid model with irregular boundary $\mathbf{R}, \tilde{\mathbf{Q}}, \tilde{\mathbf{Q}}_{\mathbf{i r}}^{(\mathbf{0})}$.

In the rest of the section we show that the sojourn time distribution can also be obtained from the solution of the fluid model defined by $\left(\mathbf{R}, \tilde{\mathbf{Q}}, \tilde{\mathbf{Q}}^{(\mathbf{0})}=\tilde{\mathbf{Q}}\right)$. 
Theorem 8. The stationary probability density function of the sojourn time of a fluid queue with independent input and output processes and boundary-continuous output policy is given by

$$
v_{\text {cont }}(x)=\frac{1}{\lambda} \tilde{\pi}(x)\left(\mathbf{R}^{(\mathbf{i n})} \otimes \mathbf{I}\right) \mathbb{1}=\frac{1}{\lambda} \tilde{\beta} e^{\tilde{\mathbf{K}} x} \mathbf{B}\left(\mathbf{R}^{(\mathbf{i n})} \otimes \mathbf{I}\right) \mathbb{1},
$$

where $\tilde{\pi}(x), \tilde{\beta}, \tilde{\mathbf{K}}$ and $\tilde{\mathbf{B}}$ are the parameters of the solution to the fluid model defined by $\left(\mathbf{R}, \tilde{\mathbf{Q}}, \tilde{\mathbf{Q}}^{(\mathbf{0})}=\tilde{\mathbf{Q}}\right)$ in (51).

Before proving the theorem three lemmas are introduced. The first lemma is a rather technical one.

Lemma 1. The following equality holds:

$$
\mathbf{K B}\left(\mathbf{R}^{(\text {in })} \otimes \mathbf{R}^{(\text {out })}\right) \mathbb{1}=\tilde{\mathbf{K}} \mathbf{B}\left(\mathbf{R}^{(\text {in })} \otimes \mathbf{I}\right) \mathbb{1} .
$$

Proof. We provide the proof only for $\mathcal{S}_{0}=\emptyset$ for simplicity (however, it is valid for the general case as well, but the cumbersome general proof is neglected here). Starting with (15) and expressing $\tilde{\mathbf{Q}}$ as $\tilde{\mathbf{Q}}=\mathbf{Q}\left(\mathbf{I} \otimes \mathbf{R}^{(\text {out })}\right)+\left(\mathbf{I} \otimes \mathbf{Q}^{(\text {out })}\right) \mathbf{R}$ gives

$$
\tilde{\mathbf{K}} \mathbf{B R}=\mathbf{B Q}\left(\mathbf{I} \otimes \mathbf{R}^{(\text {out })}\right)+\mathbf{B}\left(\mathbf{I} \otimes \mathbf{Q}^{(\text {out })}\right) \mathbf{R},
$$

which, making use of $\mathbf{B Q}=\mathbf{K B R}$ (based on (15) again) further transforms to

$$
\tilde{\mathbf{K B R}}=\mathbf{K B R}\left(\mathbf{I} \otimes \mathbf{R}^{(\text {out })}\right)+\mathbf{B}\left(\mathbf{I} \otimes \mathbf{Q}^{(\text {out })}\right) \mathbf{R} .
$$

Since $\mathcal{S}_{0}=\emptyset$ is assumed, $\mathbf{R}$ cancels at both sides. Finally, post-multiplying the equation by $\left(\mathbf{R}^{(\mathbf{i n})} \otimes \mathbf{I}\right)$ we get

$$
\tilde{\mathbf{K}} \mathbf{B}\left(\mathbf{R}^{(\text {in) }} \otimes \mathbf{I}\right)=\mathbf{K B}\left(\mathbf{R}^{(\text {in })} \otimes \mathbf{R}^{(\text {out })}\right)+\mathbf{B}\left(\mathbf{R}^{(\text {in) }} \otimes \mathbf{Q}^{(\text {out })}\right),
$$

that, recognizing $\left(\mathbf{R}^{(\mathbf{i n})} \otimes \mathbf{Q}^{(\text {out })}\right) \mathbb{1}=0$ provides (65).

In the next two lemmas we derive relations for the initial vector and the probability mass vector at 0 belonging to the following three fluid models

- for the queue length process $\left(\mathbf{Q}, \mathbf{R}, \mathbf{Q}^{(\mathbf{0})}=\mathbf{Q}\right)$, denoted by $\beta_{\text {cont }}$ and $p_{\text {cont }}$;

- for the special fluid model with irregular zero level given by $\left(\mathbf{R}, \tilde{\mathbf{Q}}, \tilde{\mathbf{Q}}_{\mathbf{i r}}^{(\mathbf{0})}\right)$, denoted by $\tilde{\beta}_{i r}$ and $\tilde{p}_{i r}$;

- for the special fluid model with regular zero level given by $\left(\mathbf{R}, \tilde{\mathbf{Q}}, \tilde{\mathbf{Q}}^{(\mathbf{0})}=\tilde{\mathbf{Q}}\right)$, denoted by $\tilde{\beta}$ and $\tilde{p}$.

We need these relations to translate the results of Theorem 7, which relies on $\tilde{\beta}_{i r}$ and $\tilde{p}_{i r}$ to a form that relies on $\tilde{\beta}$ and $\tilde{p}$ instead, since several fluid model solver implementations support only the regular boundary and it also allows the direct comparison of the boundary-proportional and the boundary-continuous cases.

The next lemma relates $\beta_{\text {cont }}, p_{\text {cont }}$ and $\tilde{\beta}, \tilde{p}$.

Lemma 2. The relations between vectors $\beta_{\text {cont }}, p_{\text {cont }}$ and $\tilde{\beta}, \tilde{p}$ are

$$
\begin{aligned}
& \beta_{\text {cont }}=\tilde{\beta}(-\tilde{\mathbf{K}})^{-1}(-\mathbf{K}), \\
& p_{\text {cont }}=\tilde{p} .
\end{aligned}
$$

Proof. The boundary equations belonging to the queue length process are (see (16))

$$
\beta_{\text {cont }} \mathbf{B R}=p_{\text {cont }} \mathbf{Q} .
$$


We are going to show that $\alpha \cdot \tilde{\beta}(-\tilde{\mathbf{K}})^{-1}(-\mathbf{K})$ and $\alpha \cdot \tilde{p}$ satisfy this equation. Substituting them into (71) gives

$$
\alpha \cdot \tilde{\beta}(-\tilde{\mathbf{K}})^{-1}(-\mathbf{K}) \mathbf{B R}=\alpha \cdot \tilde{p} \mathbf{Q},
$$

which, exploiting that $\mathbf{K B R}=\mathbf{B Q}$ based on $(15)$ and writing $(-\tilde{\mathbf{K}})^{-1}$ as an integral transforms to

$$
0=\tilde{\beta}(-\tilde{\mathbf{K}})^{-1} \mathbf{B Q}+\tilde{p} \mathbf{Q}=\left(\tilde{p}+\int_{0}^{\infty} \tilde{\beta} e^{\tilde{\mathbf{K}} x} \mathbf{B} d x\right) \mathbf{Q}
$$

Observe that the term in the big parenthesis is $\tilde{\xi}$, the stationary probability vector of $\tilde{\mathbf{Q}}$. However, looking at the definition of $\tilde{\mathbf{Q}}$ it can be easily checked that $\tilde{\xi}=\xi^{(i n)} \otimes \xi^{(\text {out })}=\xi$, thus (73) holds.

It remains to determine the normalization constant $\alpha$.

$$
\begin{aligned}
1 & =p_{\text {cont }} \mathbb{1}+\int_{0}^{\infty} \pi_{\text {cont }}(x) \mathbb{1} d x=p_{\text {cont }} \mathbb{1}+\int_{0}^{\infty} \beta_{\text {cont }} e^{\mathbf{K} x} \mathbf{B} \mathbb{1} d x \\
& =\alpha\left(\tilde{p} \mathbb{1}+\int_{0}^{\infty} \tilde{\beta}(-\tilde{\mathbf{K}})^{-1}(-\mathbf{K}) e^{\mathbf{K} x} \mathbf{B} \mathbb{1} d x\right)=\alpha\left(\tilde{p} \mathbb{1}+\tilde{\beta}(-\tilde{\mathbf{K}})^{-1}(-\mathbf{K})(-\mathbf{K})^{-1} \mathbf{B} \mathbb{1} d x\right) \\
& =\alpha\left(\tilde{p} \mathbb{1}+\int_{0}^{\infty} \tilde{\pi}(x) \mathbb{1} d x\right)=\alpha,
\end{aligned}
$$

which proves the lemma.

The last lemma needed to prove Theorem 8 relates $\beta_{\text {cont }}, p_{\text {cont }}$ and $\tilde{\beta}_{i r}, \tilde{p}_{i r}$.

Lemma 3. The relations between vectors $\beta_{\text {cont }}, p_{\text {cont }}$ and $\tilde{\beta}_{\text {ir }}, \tilde{p}_{\text {ir }}$ are

$$
\begin{aligned}
& \beta_{\text {cont }}=\frac{\lambda}{c_{i r}} \tilde{\beta}_{i r}, \\
& p_{\text {cont }}=\frac{\lambda}{c_{i r}} \tilde{p}_{i r}\left(\mathbf{I} \otimes \mathbf{R}^{(\text {out })}\right) .
\end{aligned}
$$

Proof. Let us write the boundary equations corresponding to the queue length process again as (see (16))

$$
\beta_{\text {cont }} \mathbf{B R}=p_{\text {cont }} \mathbf{Q} .
$$

Now we show that $\alpha \cdot \tilde{\beta}_{i r}$ and $\alpha \cdot \tilde{p}_{i r}\left(\mathbf{I} \otimes \mathbf{R}^{(\text {out })}\right)$ satisfy this equation. Substituting them into (77) gives

$$
\alpha \cdot \tilde{\beta}_{i r} \mathbf{B R}=\alpha \cdot \tilde{p}_{i r}\left(\mathbf{I} \otimes \mathbf{R}^{(\text {out })}\right) \mathbf{Q}
$$

which holds since $\left(\mathbf{I} \otimes \mathbf{R}^{(\text {out })}\right) \mathbf{Q}=\tilde{\mathbf{Q}}_{\mathbf{i r}}^{(\mathbf{0})}$ and $\tilde{\beta}_{i r} \mathbf{B R}=\tilde{p}_{i r} \tilde{\mathbf{Q}}_{\mathbf{i r}}^{(\mathbf{0})}$ is the boundary equation belonging to the special fluid model with irregular level zero behavior, characterized by $\left(\mathbf{R}, \tilde{\mathbf{Q}}, \tilde{\mathbf{Q}}_{\mathbf{i r}}^{(\mathbf{0})}\right)$.

The normalization constant $\alpha$ has to be set to ensure that

$$
\begin{aligned}
1 & =p_{\text {cont }} \mathbb{1}+\int_{0}^{\infty} \pi_{\text {cont }}(x) \mathbb{1} d x=p_{\text {cont }} \mathbb{1}+\int_{0}^{\infty} \beta_{\text {cont }} e^{\mathbf{K} x} \mathbf{B} \mathbb{1} d x \\
& =\alpha\left(\tilde{p}_{\text {ir }}\left(\mathbf{I} \otimes \mathbf{R}^{(\text {out })}\right)+\tilde{\beta}_{i r}(-\mathbf{K})^{-1} \mathbf{B}\right) \mathbb{1}
\end{aligned}
$$

holds.

Let us now go back to (63) and transform it as

$$
\begin{aligned}
c_{i r} & =\tilde{p}_{i r}\left(\mathbf{R}^{(\mathbf{i n})} \otimes \mathbf{R}^{(\text {out })}\right) \mathbb{1}+\underbrace{\tilde{\beta}_{i r}(-\tilde{\mathbf{K}})^{-1} \mathbf{B}\left(\mathbf{R}^{(\mathbf{i n})} \otimes \mathbf{R}^{(\text {out })}\right) \mathbb{1}}_{\tilde{\beta}_{i r}(-\mathbf{K})^{-1} \mathbf{B}\left(\mathbf{R}^{(\mathbf{i n})} \otimes \mathbf{I}\right) \mathbb{1}} \\
& =\left(\tilde{p}_{i r}\left(\mathbf{I} \otimes \mathbf{R}^{(\text {out })}\right)+\tilde{\beta}_{i r}(-\mathbf{K})^{-1} \mathbf{B}\right)\left(\mathbf{R}^{(\mathbf{i n})} \otimes \mathbf{I}\right) \mathbb{1},
\end{aligned}
$$

where we applied Lemma 1. Observe that the large parenthesis equals to $p_{\text {cont }} / \alpha+\int_{0}^{\infty} \beta_{\text {cont }}(-\mathbf{K})^{-1} \mathbf{B} / \alpha=$ $\xi / \alpha$. Since $\xi\left(\mathbf{R}^{(\mathbf{i n})} \otimes \mathbf{I}\right) \mathbb{1}=\lambda$, we have that $c_{i r}=\lambda / \alpha$, from which $\alpha=\lambda / c_{i r}$ follows. 
Finally, we can now prove Theorem 8.

Proof (Proof of Theorem 8). From Lemma 2 and 3 we have that

$$
\tilde{\beta}_{i r}=\frac{c_{i r}}{\lambda} \tilde{\beta}(-\tilde{\mathbf{K}})^{-1}(-\mathbf{K}) .
$$

Utilizing the commuting property of matrices $\mathbf{K}$ and $\tilde{\mathbf{K}}(48)$ and the relations between vectors $\tilde{\beta}_{\text {and }} \tilde{\beta}_{\text {ir }}$ given by (80) and the result of Lemma 1 yields

$$
\begin{aligned}
v_{\text {cont }}(x) & =\frac{1}{c_{\text {ir }}} \tilde{\beta}_{\text {ir }} e^{\tilde{\mathbf{K}} x} \mathbf{B}\left(\mathbf{R}^{(\mathbf{i n})} \otimes \mathbf{R}^{(\text {out })}\right) \mathbb{1}=\frac{1}{\lambda} \tilde{\beta}(-\tilde{\mathbf{K}})^{-1}(-\mathbf{K}) e^{\tilde{\mathbf{K}} x} \mathbf{B}\left(\mathbf{R}^{(\mathbf{i n})} \otimes \mathbf{R}^{(\text {out })}\right) \mathbb{1} \\
& =\frac{1}{\lambda} \tilde{\beta} e^{\tilde{\mathbf{K}} x}(-\tilde{\mathbf{K}})^{-1}(-\mathbf{K}) \mathbf{B}\left(\mathbf{R}^{(\mathbf{i n})} \otimes \mathbf{R}^{(\text {out })}\right) \mathbb{1}=\frac{1}{\lambda} \tilde{\beta} e^{\tilde{\mathbf{K}} x}\left(\mathbf{R}^{(\mathbf{i n})} \otimes \mathbf{I}\right) \mathbb{1} .
\end{aligned}
$$

We end this section by recognizing that the sojourn time has a phase-type representation in the boundarycontinuous case as well.

Theorem 9. The sojourn time of a fluid drop is phase-type distributed with

$$
P\left(\mathcal{V}_{\text {cont }} \leq t\right)=1-z_{\text {cont }} e^{\mathbf{z}_{\text {cont }} \cdot t} \mathbb{1},
$$

where

$$
\begin{aligned}
z_{\text {cont }} & =\mathbb{1}^{T}\left(\mathbf{R}^{(\text {in })^{T}} \otimes \mathbf{I}\right) \frac{1}{\lambda} \mathbf{B}^{T} \boldsymbol{\Delta}, \\
\mathbf{Z}_{\text {cont }} & =\boldsymbol{\Delta}^{-1} \tilde{\mathbf{K}}^{T} \boldsymbol{\Delta},
\end{aligned}
$$

and $\boldsymbol{\Delta}=\operatorname{diag}\left\langle\tilde{\beta}(-\tilde{\mathbf{K}})^{-1}\right\rangle$.

Proof. The proof follows the same steps as the proof of Theorem 6 .

Notice that $\mathbf{Z}_{\text {cont }}=\mathbf{Z}_{\text {prop }}$, thus the phase-type representation of the sojourn times for the two boundary behaviors considered differ only in the initial vector.

\section{Summary}

We have derived the sojourn time distribution of fluid queues with both dependent and independent input and output processes.

The derivations in the dependent case are similar to the ones provided by Ozawa in [4] for the discrete queues, resulting in an order $N \cdot N_{+}$phase-type distribution for the sojourn time.

The main contribution of the paper is, however, the case when the input and output processes are independent. We proved that the sojourn time distribution is related to the stationary distribution $\tilde{\pi}(x)$ of the fluid model (with regular boundary) defined by

$$
\frac{d}{d x} \tilde{\pi}(x)\left(\mathbf{R}^{(\text {in })} \otimes \mathbf{I}-\mathbf{I} \otimes \mathbf{R}^{(\text {out })}\right)=\tilde{\pi}(x)\left(\mathbf{Q}^{(\text {in })} \otimes \mathbf{R}^{(\text {out })}+\mathbf{R}^{(\text {in })} \otimes \mathbf{Q}^{(\text {out })}\right),
$$

which can be solved in a numerically efficient way e.g., by matrix-analytic methods [10].

From $\tilde{\pi}(x)$, the stationary density of the sojourn time is obtained as

$$
v_{\text {prop }}(x)=\frac{1}{\lambda \mu} \tilde{\pi}(x)\left(\mathbf{R}^{(\text {in })} \otimes \mathbf{R}^{(\text {out })}\right) \mathbb{1}
$$

in the boundary-proportional case, and it is

$$
v_{\text {cont }}(x)=\frac{1}{\lambda} \tilde{\pi}(x)\left(\mathbf{R}^{(\mathbf{i n})} \otimes \mathbf{I}\right) \mathbb{1}
$$


in the boundary-continuous case. Both cases lead to size $N_{+}$phase-type representations.

Finally, the introduction of the age process approach for discrete queues turned out to be useful not only in the sojourn time analysis, but also in the efficient solution of multi-type queues and queues with impatient customers. We believe that the age process for continuous queues, that we defined and analyzed as a technical aid for the sojourn time analysis, will be a useful tool for obtaining similar results for continuous queues in the future.

[1] L. Kleinrock, Theory, Volume 1, Queueing Systems, Wiley-Interscience, 1975.

[2] D. Anick, D. Mitra, M. M. Sondhi, Stochastic Theory of a Data-Handling System with Multiple Sources, Bell System Technical Journal 61 (8) (1982) 1871-1894.

[3] M. Neuts, Matrix Geometric Solutions in Stochastic Models, Johns Hopkins University Press, Baltimore, 1981.

[4] T. Ozawa, Sojourn time distributions in the queue defined by a general QBD process, Queueing Syst. Theory Appl. 53 (4) (2006) 203-211, ISSN 0257-0130, doi:10.1007/s11134-006-7651-3, URL http://dx.doi.org/10.1007/s11134-006-7651-3.

[5] B. Sengupta, The semi-Markovian queue: theory and applications, Stochastic Models 6 (3) (1990) 383-413.

[6] B. Sengupta, Markov processes whose steady state distribution is matrix-exponential with an application to the GI/GH/1 queue, Advances in Applied Probability 21 (1) (1989) 159-180.

[7] B. Van Houdt, A Phase-Type Representation for the Queue Length Distribution of a Semi-Markovian Queue., in: QEST, IEEE Computer Society, 2010.

[8] V. Ramaswami, Matrix analytic methods for stochastic fluid flows, in: Teletraffic Engineering in a Competitive World Proc. of the 16th International Teletraffic Congress (ITC 16), Elsevier Science B.V., 1019-1030, 1999.

[9] V. G. Kulkarni, Fluid models for single buffer systems, Frontiers in queueing: Models and applications in science and engineering 321 (1997) 338.

[10] S. Soares, G. Latouche, Further results on the similarity between fluid queues and QBDs, in: Proceedings of the 4th international conference on matrix-analytic methods, 89-106, 2002.

[11] V. G. Kulkarni, V. F. Nicola, K. S. Trivedi, On modelling the performance and reliability of multimode computer systems, Journal of Systems and Software 6 (1) (1986) 175-182.

[12] A. J. Laub, Matrix analysis for scientists and engineers, Siam, 2005.

[13] H. Masuyama, T. Takine, Multiclass Markovian fluid queues, Queueing Systems 56 (3-4) (2007) 143-155.

[14] G. Horváth, M. Telek, B. Van Houdt, Commuting matrices in the sojourn time analysis of MAP/MAP/1 queues, in: Matrix Analytic Methods in Stochastic Models (MAM8), 75-83, 2014.

\section{Appendix A. Proof of Theorem 4}

PROOF.

- $\tilde{\Psi}=\Psi$

We prove this equality by probabilistic arguments. Let us investigate the transition probability matrix of phases between the beginning and the end of the busy period of the queue $(\mathcal{X}>0)$. This quantity can be obtained by two different ways.

- Based on the fluid level process. Matrix $\Psi$ corresponding to the fluid model characterized by $(\mathbf{Q}, \mathbf{R})$ holds exactly these probabilities by definition.

- Based on the age process. Although the trajectory of the age and the queue length process are different in general, they coincide when the busy period starts and when it ends. Furthermore, due to the nature of the age process, the phase of the age process and the one of the queue length process are the same when the busy period begins. Up to the time instant when the queue gets empty again both the input and the output process evolve the same amount as the input and output process of the queue length process. This is because during a busy period the same amount of fluid arrives and leaves the queue both in the age process and the queue length process. Thus, the phase transition probability matrix of the age process must be $\boldsymbol{\Psi}$ as well.

According to the proof of Theorem 5 (see Appendix Appendix B) the fluid model characterized by $(\mathbf{R}, \tilde{\mathbf{Q}})$ differs from the one of the age process only in a matrix multiplier $\mathbf{R}^{(\mathbf{i n})} \otimes \mathbf{R}^{\text {(out) }}$, however, such a scaling does not have any effect on the state transition probability matrix we need. Consequently, matrix $\tilde{\boldsymbol{\Psi}}$ corresponding to fluid model $(\mathbf{R}, \tilde{\mathbf{Q}})$ equals to $\Psi^{5}$.

\footnotetext{
${ }^{5}$ The discrete counterpart of this result can be found in [14]. If we post-multiply the equality (42) in [14] by $\mathbf{I} \otimes \mathbf{S}_{\mathbf{1}}$, we get matrix $\mathbf{G}$ at the left hand side (which plays the role of $\boldsymbol{\Psi}$ in our case), and we get the probability transition matrix for the phases of the age process at the right hand side (which is $\tilde{\boldsymbol{\Psi}}$ in this paper).
} 
- $\tilde{\mathbf{B}}=\mathbf{B}$

Looking at the definitions of $\mathbf{B}$ and $\tilde{\mathbf{B}}$ (see (10)) we have that $\tilde{\mathbf{R}}=\mathbf{R}$ and $\tilde{\mathbf{\Psi}}=\mathbf{\Psi}$, thus it suffices to show that $\mathbf{Q}_{+\mathbf{0}}\left(-\mathbf{Q}_{\mathbf{0 0}}\right)^{-1}=\tilde{\mathbf{Q}}_{+\mathbf{0}}\left(-\tilde{\mathbf{Q}}_{\mathbf{0 0}}\right)^{-1}$ and $\mathbf{Q}_{-\mathbf{0}}\left(-\mathbf{Q}_{\mathbf{0 0}}\right)^{-1}=\tilde{\mathbf{Q}}_{-\mathbf{0}}\left(-\tilde{\mathbf{Q}}_{\mathbf{0 0}}\right)^{-1}$. Making use of the structure of matrix $\tilde{\mathbf{Q}}$ according to (45) gives

$$
\tilde{\mathbf{Q}}_{+\mathbf{0}}\left(-\tilde{\mathbf{Q}}_{\mathbf{0 0}}\right)^{-1}=\left(\mathbf{Q}^{(\mathrm{in})} \otimes \mathbf{R}^{(\text {out })}+\mathbf{R}^{(\mathrm{in})} \otimes \mathbf{Q}^{(\text {out })}\right)_{+0}\left(\mathbf{Q}^{(\text {in })} \otimes \mathbf{R}^{(\text {out })}+\mathbf{R}^{(\mathrm{in})} \otimes \mathbf{Q}^{(\text {out })}\right)_{00}^{-1},
$$

where the $\mathbf{R}^{(\mathbf{i n})}$ and $\mathbf{R}^{(\text {out })}$ terms cancel since $\mathbf{R}^{(\text {out })}{ }_{i}=\mathbf{R}^{(\mathbf{i n})}{ }_{i}$ holds for zero states, leading to $\mathbf{Q}_{+\mathbf{0}}\left(-\mathbf{Q}_{\mathbf{0 0}}\right)^{-1}$ that provides the theorem.

- $\left(\mathbf{R}^{\bullet-1} \mathbf{Q}^{\bullet}\right)\left(\mathbf{R}^{\bullet-1} \tilde{\mathbf{Q}}^{\bullet}\right)=\left(\mathbf{R}^{\bullet-1} \tilde{\mathbf{Q}}^{\bullet}\right)\left(\mathbf{R}^{\bullet-1} \mathbf{Q}^{\bullet}\right)$

We provide the proof only for the case when $\mathcal{S}_{0}=\emptyset$. The theorem holds for the general case as well, but the increased technical complexity makes the proof long.

When $\mathcal{S}_{0}=\emptyset$ we have that $\mathbf{Q}^{\bullet}=\mathbf{Q}, \tilde{\mathbf{Q}}^{\bullet}=\tilde{\mathbf{Q}}$ and $\mathbf{R}^{\bullet}=\mathbf{R}$. From the definitions of $\mathbf{Q}$ (see (39)), $\mathbf{R}$ (see (38)) and $\tilde{\mathbf{Q}}$ (see (45)) we can express matrix $\tilde{\mathbf{Q}}$ in two different ways. First, it can be expressed by

$$
\tilde{\mathbf{Q}}=\left(\mathbf{R}^{(\mathbf{i n})} \otimes \mathbf{I}\right) \mathbf{Q}-\mathbf{R}\left(\mathbf{Q}^{(\mathbf{i n})} \otimes \mathbf{I}\right),
$$

and it can be also expressed by

$$
\tilde{\mathbf{Q}}=\mathbf{Q}\left(\mathbf{R}^{(\mathbf{i n})} \otimes \mathbf{I}\right)-\left(\mathbf{Q}^{(\mathbf{i n})} \otimes \mathbf{I}\right) \mathbf{R} .
$$

Now let us pre-multiply (A.2) by $\mathbf{Q R}^{-1}$ yielding

$$
\begin{aligned}
\mathbf{Q} \mathbf{R}^{-1} \tilde{\mathbf{Q}} & =\mathbf{Q} \underbrace{\mathbf{R}^{-1}\left(\mathbf{R}^{(\mathbf{i n})} \otimes \mathbf{I}\right)}_{\text {commute }} \mathbf{Q}-\underbrace{\mathbf{Q}\left(\mathbf{Q}^{(\mathbf{i n})} \otimes \mathbf{I}\right)}_{\text {commute }} \\
& =(\underbrace{\mathbf{Q}\left(\mathbf{R}^{(\mathbf{i n})} \otimes \mathbf{I}\right)-\left(\mathbf{Q}^{(\mathbf{i n})} \otimes \mathbf{I}\right) \mathbf{R}}_{=\tilde{\mathbf{Q}} \text {, due to (A.3) }}) \mathbf{R}^{-1} \mathbf{Q}=\tilde{\mathbf{Q}} \mathbf{R}^{-1} \mathbf{Q},
\end{aligned}
$$

which completes the proof.

- $\mathbf{K} \tilde{\mathbf{K}}=\tilde{\mathbf{K}} \mathbf{K}$

Let us first recall the definitions of matrices $\mathbf{K}$ and $\tilde{\mathbf{K}}$, making use of $\tilde{\boldsymbol{\Psi}}=\boldsymbol{\Psi}$ right away:

$$
\begin{aligned}
\mathbf{K} & =\mathbf{R}_{+}{ }^{-1} \mathbf{Q}_{++}^{\bullet}+\mathbf{\Psi}\left|\mathbf{R}_{-}\right|^{-1} \mathbf{Q}_{-+}^{\bullet}, \\
\tilde{\mathbf{K}} & =\mathbf{R}_{+}{ }^{-1} \tilde{\mathbf{Q}}_{++}^{\bullet}+\boldsymbol{\Psi}\left|\mathbf{R}_{-}\right|^{-1} \tilde{\mathbf{Q}}_{-+}^{\bullet} .
\end{aligned}
$$

Multiplying them gives

$$
\begin{aligned}
\mathbf{K} \tilde{\mathbf{K}} & =\mathbf{R}_{+}{ }^{-1} \mathbf{Q}_{++}^{\bullet} \mathbf{R}_{+}{ }^{-1} \tilde{\mathbf{Q}}_{++}^{\bullet}+\mathbf{R}_{+}{ }^{-1} \mathbf{Q}_{++}^{\bullet} \boldsymbol{\Psi}\left|\mathbf{R}_{-}\right|^{-1} \tilde{\mathbf{Q}}_{-+}^{\bullet} \\
& +\boldsymbol{\Psi}\left|\mathbf{R}_{-}\right|^{-1} \mathbf{Q}_{-+}^{\bullet} \mathbf{R}_{+}{ }^{-1} \tilde{\mathbf{Q}}_{++}^{\bullet}+\Psi\left|\mathbf{R}_{-}\right|^{-1} \mathbf{Q}_{-+}^{\bullet} \boldsymbol{\Psi}\left|\mathbf{R}_{-}\right|^{-1} \tilde{\mathbf{Q}}_{-+}^{\bullet}
\end{aligned}
$$

From (7) we have that $\boldsymbol{\Psi}\left|\mathbf{R}_{-}\right|^{-1} \mathbf{Q}_{-+}^{\bullet} \mathbf{\Psi}+\mathbf{R}_{+}{ }^{-1} \mathbf{Q}_{++}^{\bullet} \mathbf{\Psi}=-\mathbf{R}_{+}{ }^{-1} \mathbf{Q}_{+-}^{\bullet}-\boldsymbol{\Psi}\left|\mathbf{R}_{-}\right|^{-1} \mathbf{Q}_{--}^{\bullet}$, yielding

$$
\begin{aligned}
\mathbf{K} \tilde{\mathbf{K}} & =\mathbf{R}_{+}{ }^{-1} \mathbf{Q}_{++}^{\bullet} \mathbf{R}_{+}{ }^{-1} \tilde{\mathbf{Q}}_{++}^{\bullet}-\mathbf{R}_{+}{ }^{-1} \mathbf{Q}_{+-}^{\bullet}\left|\mathbf{R}_{-}\right|^{-1} \tilde{\mathbf{Q}}_{-+}^{\bullet} \\
& +\boldsymbol{\Psi}\left|\mathbf{R}_{-}\right|^{-1} \mathbf{Q}_{-+}^{\bullet} \mathbf{R}_{+}{ }^{-1} \tilde{\mathbf{Q}}_{++}^{\bullet}-\boldsymbol{\Psi}\left|\mathbf{R}_{-}\right|^{-1} \mathbf{Q}_{--}^{\bullet}\left|\mathbf{R}_{-}\right|^{-1} \tilde{\mathbf{Q}}_{-+}^{\bullet},
\end{aligned}
$$

which, exploiting that the following 2 relations hold due to (47)

$$
\begin{aligned}
& \mathbf{R}_{+}{ }^{-1} \tilde{\mathbf{Q}}_{++}^{\bullet} \mathbf{R}_{+}{ }^{-1} \mathbf{Q}_{++}^{\bullet}-\mathbf{R}_{+}{ }^{-1} \mathbf{Q}_{+-}^{\bullet} \mathbf{R}_{-}{ }^{-1} \tilde{\mathbf{Q}}_{-+}^{\bullet}=\mathbf{R}_{+}{ }^{-1} \mathbf{Q}_{++}^{\bullet} \mathbf{R}_{+}{ }^{-1} \tilde{\mathbf{Q}}_{++}^{\bullet}-\mathbf{R}_{+}{ }^{-1} \tilde{\mathbf{Q}}_{+-}^{\bullet} \mathbf{R}_{-}{ }^{-1} \mathbf{Q}_{-+}^{\bullet}, \\
& \mathbf{R}_{-}{ }^{-1} \tilde{\mathbf{Q}}_{-+}^{\bullet} \mathbf{R}_{+}{ }^{-1} \mathbf{Q}_{++}^{\bullet}-\mathbf{R}_{-}{ }^{-1} \mathbf{Q}_{--}^{\bullet} \mathbf{R}_{-}{ }^{-1} \tilde{\mathbf{Q}}_{-+}^{\bullet}=\mathbf{R}_{-}{ }^{-1} \mathbf{Q}_{-+}^{\bullet} \mathbf{R}_{+}{ }^{-1} \tilde{\mathbf{Q}}_{++}^{\bullet}-\mathbf{R}_{-}{ }^{-1} \tilde{\mathbf{Q}}_{--}^{\bullet} \mathbf{R}_{-}{ }^{-1} \mathbf{Q}_{-+}^{\bullet},
\end{aligned}
$$


equals to

$$
\begin{aligned}
\mathbf{K} \tilde{\mathbf{K}} & =\mathbf{R}_{+}{ }^{-1} \tilde{\mathbf{Q}}_{++}^{\bullet} \mathbf{R}_{+}{ }^{-1} \mathbf{Q}_{++}^{\bullet}-\mathbf{R}_{+}{ }^{-1} \tilde{\mathbf{Q}}_{+-}^{\bullet}\left|\mathbf{R}_{-}\right|^{-1} \mathbf{Q}_{-+}^{\bullet} \\
& +\Psi\left|\mathbf{R}_{-}\right|^{-1} \tilde{\mathbf{Q}}_{-+}^{\bullet} \mathbf{R}_{+}{ }^{-1} \mathbf{Q}_{++}^{\bullet}-\boldsymbol{\Psi}\left|\mathbf{R}_{-}\right|^{-1} \tilde{\mathbf{Q}}_{--}^{\bullet}\left|\mathbf{R}_{-}\right|^{-1} \mathbf{Q}_{-+}^{\bullet}
\end{aligned}
$$

Using $\mathbf{R}_{+}{ }^{-1} \tilde{\mathbf{Q}}_{+-}^{\bullet}+\boldsymbol{\Psi}\left|\mathbf{R}_{-}\right|^{-1} \tilde{\mathbf{Q}}_{--}^{\bullet}=-\boldsymbol{\Psi}\left|\mathbf{R}_{-}\right|^{-1} \tilde{\mathbf{Q}}_{-+}^{\bullet} \boldsymbol{\Psi}-\mathbf{R}_{+}{ }^{-1} \tilde{\mathbf{Q}}_{++}^{\bullet} \boldsymbol{\Psi}$ based on the Riccati equation for $(\tilde{\mathbf{R}}, \tilde{\mathbf{Q}})$ and $\tilde{\boldsymbol{\Psi}}=\boldsymbol{\Psi}$ leads to

$$
\begin{aligned}
\mathbf{K} \tilde{\mathbf{K}} & =\mathbf{R}_{+}{ }^{-1} \tilde{\mathbf{Q}}_{++}^{\bullet} \mathbf{R}_{+}{ }^{-1} \mathbf{Q}_{++}^{\bullet}+\mathbf{R}_{+}{ }^{-1} \tilde{\mathbf{Q}}_{++}^{\bullet} \boldsymbol{\Psi}\left|\mathbf{R}_{-}\right|^{-1} \mathbf{Q}_{-+}^{\bullet} \\
& +\boldsymbol{\Psi}\left|\mathbf{R}_{-}\right|^{-1} \tilde{\mathbf{Q}}_{-+}^{\bullet} \mathbf{R}_{+}{ }^{-1} \mathbf{Q}_{++}^{\bullet}+\boldsymbol{\Psi}\left|\mathbf{R}_{-}\right|^{-1} \tilde{\mathbf{Q}}_{-+}^{\bullet} \boldsymbol{\Psi}\left|\mathbf{R}_{-}\right|^{-1} \mathbf{Q}_{-+}^{\bullet},
\end{aligned}
$$

that equals to $\tilde{\mathbf{K}} \mathbf{K}$.

\section{Appendix B. Proof of Theorem 5}

We present the proof in two steps. In the fist step we focus on the case with positive input and output rates, and in the second step we generalize the proof for the non-negative cases.

Appendix B.1. The Proof of Theorem 5 with nonzero $r^{(i n)}$ and $r^{(\text {out })}$ rates

First we derive the set of differential and boundary equations which characterize the age process of fluid drops.

Proof. We assume that fluid arrive to the buffer in drops of size $D$ (which we let tend to zero later).

As long as the input Markov chain stays in state $i$, that is $\mathcal{Z}^{(i n)}(t)=i$, drops arrive with inter-arrival time $D / r_{i}^{(i n)}$. At the state transitions of the Markov chain the inter-arrival time is different, denoted by $t^{*}$, defined by $\int_{0}^{t^{*}} r_{\mathcal{Z}^{(i n)}(t)}^{(i n)} d t=D$. If $\mathcal{Z}^{(i n)}(t)=k$ and $\mathcal{Z}^{(i n)}\left(t+D / r_{k}^{(i n)}\right)=i$ such that the state transition occurs at $t+y$ we have

$$
t^{*}=I_{k i}^{(i n)}(y)=\frac{D}{r_{i}^{(i n)}}+y\left(1-\frac{r_{k}^{(i n)}}{r_{i}^{(i n)}}\right)
$$

Note that $I_{k i}^{(i n)}(0)=D / r_{i}^{(i n)}$ and $I_{k i}^{(i n)}\left(D / r_{k}^{(i n)}\right)=D / r_{k}^{(i n)}$.

Similarly, drops depart from the buffer with inter-departure time $D / r_{j}^{(o u t)}$ as long as the output Markov chain stays in state $j$, that is $\mathcal{Z}^{(\text {out })}(t)=j$. At the state transitions of the output Markov chain interdeparture time is defined by $\int_{0}^{t^{*}} r_{\mathcal{Z}^{(o u t)}(t)}^{(\text {out })} d t=D$. If $\mathcal{Z}^{(\text {out })}(t)=\ell$ and $\mathcal{Z}^{(\text {out })}\left(t+D / r_{\ell}^{(\text {out })}\right)=j$ such that the state transition occurs at $t+y$ then

$$
t^{*}=I_{\ell j}^{(\text {out })}(y)=\frac{D}{r_{j}^{(o u t)}}+y\left(1-\frac{r_{\ell}^{(o u t)}}{r_{j}^{(o u t)}}\right) .
$$

Again we have $I_{\ell j}^{(\text {out })}(0)=D / r_{j}^{(\text {out })}$ and $I_{\ell j}^{(\text {out })}\left(D / r_{\ell}^{(\text {in })}\right)=D / r_{\ell}^{(o u t)}$.

The age process is continuously increasing at rate of one during the service of a drop and jumps down at the service completion instant (see Figure B.1). The size of the downward jump is the inter-arrival time between the drop under service and the next drop if the buffer is not empty. We investigate the age process just before and just after drop departure events and define the following two probabilities respectively

$$
\begin{aligned}
& f_{i j}(t, x)=P\left(\mathcal{Z}^{(i n)}(t)=i, \mathcal{Z}^{(\text {out })}(t)=j, \mathcal{A}\left(t^{+}\right)=x\right), \quad x>0, \\
& g_{i j}(t, x)=P\left(\mathcal{Z}^{(i n)}(t)=i, \mathcal{Z}^{(\text {out })}(t)=j, \mathcal{A}\left(t^{-}\right)=x\right), \quad x>0 .
\end{aligned}
$$




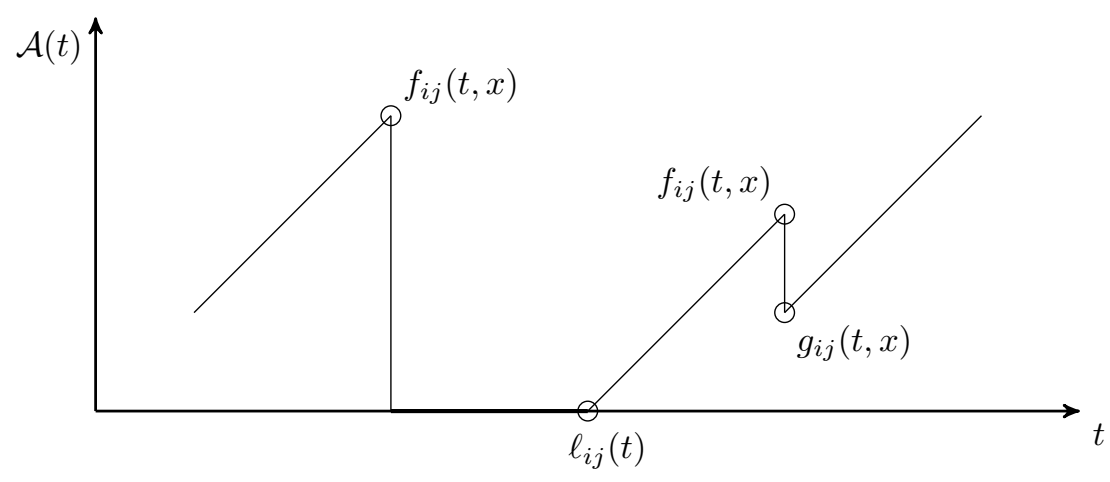

Figure B.1: Age process with positive rates

To characterize the age process when the buffer is empty we further define

$$
\ell_{i j}(t)=P\left(\mathcal{Z}^{(i n)}(t)=i, \mathcal{Z}^{(\text {out })}(t)=j, \mathcal{A}\left(t^{+}\right)=0\right)
$$

where $t^{+}$is the time instant of a drop arrival into empty buffer. For $f_{i j}(t, x)$ we have

$$
\begin{aligned}
f_{i j}(t, x) & =\left(1+q_{i i}^{(i n)} \frac{D}{r_{i}^{(i n)}}\right) g_{i j}\left(t, x+\frac{D}{r_{i}^{(i n)}}\right)+ \\
& +\sum_{k \in \mathcal{S}^{(i n)}, k \neq i} \int_{y=0}^{D / r_{k}^{(i n)}} \frac{q_{k i}^{(i n)}}{-q_{k k}^{(i n)}}\left(-q_{k k}^{(i n)}\right) e^{q_{k k}^{(i n)} y} g_{k j}\left(t, x+I_{k i}^{(i n)}(y)\right) d y+\sigma(D),
\end{aligned}
$$

which, considering that $I_{k i}^{(i n)}(y) \in\left(D / r_{i}^{(i n)}, D / r_{k}^{(i n)}\right)$ simplifies to

$$
f_{i j}(t, x)=\left(1+q_{i i}^{(i n)} \frac{D}{r_{i}^{(i n)}}\right) g_{i j}\left(t, x+\frac{D}{r_{i}^{(i n)}}\right)+\sum_{k \in \mathcal{S}^{(i n)}, k \neq i} q_{k i}^{(i n)} \frac{D}{r_{k}^{(i n)}} g_{k j}(t, x+\Theta(D))+\sigma(D),
$$

where $\Theta(D)$ is such that $\lim _{D \rightarrow 0} \Theta(D)=0$ and $\sigma(D)$ is such that $\lim _{D \rightarrow 0} \sigma(D) / D=0$. To derive $g_{i j}(t, x)$ we need to be careful with small $x$ values $(x \sim \Theta(D))$

$$
\begin{aligned}
& g_{i j}(t, x)=\left(1+q_{j j}^{\text {(out })} \frac{D}{r_{j}^{(\text {out })}}\right) f_{i j}\left(t-\frac{D}{r_{j}^{(\text {out })}}, x-\frac{D}{r_{j}^{\text {out })}}\right) \mathcal{I}_{\left\{x>D / r_{j}^{\text {out })}\right\}^{+}} \\
& +\left(1+q_{j j}^{(\text {out })} \frac{D}{r_{j}^{(\text {out })}}\right) \ell_{i j}\left(t-\frac{D}{r_{j}^{(o u t)}}\right) \delta\left(x-\frac{D}{r_{j}^{(\text {out })}}\right)+ \\
& +\sum_{\ell \in \mathcal{S}^{(\text {out })}, \ell \neq j} \int_{y=0}^{D / r_{\ell}^{(\text {out })}} q_{\ell j}^{(\text {out })} e^{q_{\ell \ell}^{(\text {out })} y} f_{i \ell}\left(t-I_{\ell j}^{(\text {out })}(y), x-I_{\ell j}^{(\text {out })}(y)\right) \mathcal{I}_{\left\{x>I_{\ell j}^{(\text {out })}(y)\right\}} d y+ \\
& +\sum_{\ell \in \mathcal{S}(\text { out }), \ell \neq j} \int_{y=0}^{D / r_{\ell}^{(o u t)}} q_{\ell j}^{(\text {out })} e^{q_{\ell \ell}^{(o u t)} y} \ell_{i \ell}\left(t-I_{\ell j}^{(\text {out })}(y)\right) \delta\left(x-I_{\ell j}^{(\text {out })}(y)\right) d y+\sigma(D),
\end{aligned}
$$

where $\delta(x)$ is the Dirac delta function. For $\ell_{i j}(t)$ we have

$$
\begin{aligned}
\ell_{i j}(t)= & \left(1+q_{i i}^{(i n)} \frac{D}{r_{i}^{(i n)}}\right) \int_{y=0}^{\frac{D}{r_{i}^{(i n)}}} g_{i j}\left(t-y, \frac{D}{r_{i}^{(i n)}}-y\right) d y+\sigma(D)+ \\
& +\sum_{k \in \mathcal{S}^{(i n)}, k \neq i} \int_{y=0}^{D / r_{k}^{(i n)}} q_{k i}^{(i n)} e^{q_{k k}^{(i n)} y} \int_{z=0}^{I_{k i}^{(i n)}(y)} g_{k j}\left(t-z, I_{k i}^{(i n)}(y)-z\right) d z d y
\end{aligned}
$$


Analysis for large $x$ values. For $x>\Theta(D)$ (B.5) simplifies to

$$
\begin{aligned}
g_{i j}(t, x)= & \left(1+q_{j j}^{\text {(out })} \frac{D}{r_{j}^{(\text {out })}}\right) f_{i j}\left(t-\frac{D}{r_{j}^{(\text {out })}}, x-\frac{D}{r_{j}^{(\text {out })}}\right)+ \\
& +\sum_{\ell \in \mathcal{S}^{\text {out })}, \ell \neq j} q_{\ell j}^{(\text {out })} \frac{D}{r_{\ell}^{(\text {out })}} f_{i \ell}(t-\Theta(D), x-\Theta(D))+\sigma(D) .
\end{aligned}
$$

Eliminating the first brackets on the right hand side of (B.4) and (B.7) gives

$$
f_{i j}(t, x)=g_{i j}\left(t, x+\frac{D}{r_{i}^{(i n)}}\right)+\sum_{k \in \mathcal{S}^{(i n)}} q_{k i}^{(i n)} \frac{D}{r_{k}^{(i n)}} g_{k j}(t, x+\Theta(D))+\sigma(D),
$$

and

$$
g_{i j}(t, x)=f_{i j}\left(t-\frac{D}{r_{j}^{(\text {out })}}, x-\frac{D}{r_{j}^{(\text {out })}}\right)+\sum_{\ell \in \mathcal{S}^{(\text {out })}} q_{\ell j}^{(\text {out })} \frac{D}{r_{\ell}^{(\text {out })}} f_{i \ell}(t-\Theta(D), x-\Theta(D))+\sigma(D) .
$$

Substituting (B.8) into (B.9) yields

$$
\begin{aligned}
g_{i j}(t, x)= & g_{i j}\left(t-\frac{D}{r_{j}^{(\text {out })}}, x-\frac{D}{r_{j}^{(\text {out })}}+\frac{D}{r_{i}^{(\text {in })}}\right)+\sum_{\ell \in \mathcal{S}^{\text {(out })}} q_{\ell j}^{(\text {out })} \frac{D}{r_{\ell}^{(\text {out })}} g_{i \ell}(t-\Theta(D), x-\Theta(D))+ \\
& +\sum_{k \in \mathcal{S}^{(i n)}} q_{k i}^{(i n)} \frac{D}{r_{k}^{(i n)}} g_{k j}(t-\Theta(D), x-\Theta(D))+\sigma(D) .
\end{aligned}
$$

Dividing both sides by $D$ and letting $D$ tend to 0 gives

$$
\begin{aligned}
& \frac{1}{D}\left(g_{i j}(t, x)-g_{i j}\left(t-\frac{D}{r_{j}^{(\text {out })}}, x-\frac{D}{r_{j}^{(\text {out })}}+\frac{D}{r_{i}^{(\text {in })}}\right)\right)= \\
& =\sum_{\ell \in \mathcal{S}^{(\text {out })}} q_{\ell j}^{(\text {out })} \frac{1}{r_{\ell}^{(\text {out })}} g_{i \ell}(t-\Theta(D), x-\Theta(D))+\sum_{k \in \mathcal{S}^{(\text {in })}} q_{k i}^{(\text {in })} \frac{1}{r_{k}^{(\text {in })}} g_{k j}(t-\Theta(D), x-\Theta(D))+\frac{\sigma(D)}{D},
\end{aligned}
$$

and

$$
\begin{aligned}
& \frac{1}{r_{j}^{(\text {out })}} \frac{\partial}{\partial t} g_{i j}(t, x)+\left(\frac{1}{r_{j}^{(\text {out })}}-\frac{1}{r_{i}^{(\text {in })}}\right) \frac{\partial}{\partial x} g_{i j}(t, x)= \\
& \sum_{\ell \in \mathcal{S}^{\text {(out })}} q_{\ell j}^{(\text {out })} \frac{1}{r_{\ell}^{(\text {out })}} g_{i \ell}(t, x)+\sum_{k \in \mathcal{S}^{(i n)}} q_{k i}^{(\text {in })} \frac{1}{r_{k}^{(i n)}} g_{k j}(t, x) .
\end{aligned}
$$

Assuming that the stationary limit exist, $\lim _{t \rightarrow \infty} g_{i j}(t, x)=g_{i j}(x)$, we have

$$
\begin{aligned}
\left(\frac{1}{r_{j}^{(\text {out })}}-\frac{1}{r_{i}^{(\text {in })}}\right) \frac{d}{d x} g_{i j}(x)= & \sum_{\ell \in \mathcal{S}_{\text {(out })}} q_{\ell j}^{(\text {out })} \frac{1}{r_{\ell}^{(\text {out })}} g_{i \ell}(x) \\
& +\sum_{k \in \mathcal{S}^{(i n)}} q_{k i}^{(i n)} \frac{1}{r_{k}^{(i n)}} g_{k j}(x), \quad i \in \mathcal{S}^{(i n)}, j \in \mathcal{S}^{(\text {out })},
\end{aligned}
$$

and the vector representation of (B.11) becomes

$$
\frac{\partial}{\partial x} g(x)\left(-\mathbf{R}^{(\mathbf{i n})^{-1}} \otimes \mathbf{I}+\mathbf{I} \otimes \mathbf{R}^{(\text {out })^{-1}}\right)=g(x)\left(\mathbf{R}^{(\mathbf{i n})^{-1}} \mathbf{Q}^{(\mathbf{i n})} \otimes \mathbf{I}+\mathbf{I} \otimes \mathbf{R}^{(\text {out })^{-1}} \mathbf{Q}^{(\text {out })}\right) .
$$


Boundary behavior. To compute the boundary behavior we approximate $I_{k i}^{(i n)}(y)$ by $\frac{D}{r_{k}^{(i n)}}\left(\right.$ B.6) and $I_{\ell j}^{(o u t)}(y)$ by $\frac{D}{r_{\ell}^{(o u t)}}$ in (B.5) hereafter. From (B.6) we obtain

$$
\begin{aligned}
\ell_{i j}(t) & =\left(1+q_{i i}^{(i n)} \frac{D}{r_{i}^{(i n)}}\right) \int_{y=0}^{\frac{D}{r_{i}^{(i n)}}} g_{i j}\left(t-y, \frac{D}{r_{i}^{(i n)}}-y\right) d y+ \\
& +\sum_{k \in \mathcal{S}^{(i n)}, k \neq i} \underbrace{\int_{y=0}^{D / r_{k}^{(i n)}} q_{k i}^{(i n)} e^{q_{k k}^{(i n)} y} d y}_{\frac{D}{r_{k}^{(i n)}} q_{k i}^{(i n)}} \int_{z=0}^{\frac{D}{r_{k}^{(i n)}}} g_{k j}\left(t-z, \frac{D}{r_{k}^{(i n)}}-z\right) d z+\sigma(D) .
\end{aligned}
$$

In this expression we have an integral of $g_{i j}(t, x)$ in the first row and the same kind integral multiplied by $D$ in the second row. In order to evaluate the second row it is enough to evaluate the integral up to the $\Theta(D)$ terms, while in case of the first row we need to evaluate the integral up to the $\sigma(D)$ terms. The $\Theta(D)$ level description of the integral is

$$
\begin{aligned}
& \int_{z=0}^{c D} g_{i j}(t-z, x-z) d x= \\
& =\underbrace{\int_{z=0}^{c D}\left(1+q_{j j}^{(\text {out })} \frac{D}{r_{j}^{(\text {out })}}\right) f_{i j}\left(t-\frac{D}{r_{j}^{(\text {out })}}-z, x-\frac{D}{r_{j}^{(\text {out })}}-z\right) \mathcal{I}_{\left\{x>D / r_{j}^{(\text {out })}\right\}} d z}_{\Theta(D)}+ \\
& +\underbrace{\int_{z=0}^{c D}\left(1+q_{j j}^{(\text {out })} \frac{D}{r_{j}^{(\text {out })}}\right) \ell_{i j}\left(t-\frac{D}{r_{j}^{(\text {out })}}-z\right) \delta\left(x-\frac{D}{r_{j}^{(\text {out })}}-z\right) d z}_{\ell_{i j}(t-x) \mathcal{I}_{\left\{0<x-D / r_{j}^{(\text {out })}<c D\right\}}+\Theta(D)}+ \\
& +\underbrace{\int_{z=0}^{c D} \sum_{\ell \in \mathcal{S}^{(\text {out })}, \ell \neq j} \int_{y=0}^{D / r_{\ell}^{(\text {out })}} q_{\ell j}^{(\text {out })} e^{q_{\ell \ell}^{(\text {out })} y} f_{i \ell}\left(t-I_{\ell j}^{(\text {out })}(y)-z, x-I_{\ell j}^{(\text {out })}(y)-z\right) \mathcal{I}_{\left\{x>I_{\ell j}^{(\text {out })}(y)\right\}} d y d z}_{\sigma(D)}+ \\
& +\underbrace{\int_{z=0}^{c D} \sum_{\ell \in \mathcal{S}^{(\text {out })}, \ell \neq j \ell \in \mathcal{S}^{(\text {out })}, \ell \neq j} \int_{y=0}^{D / r_{\ell}^{(o u t)}} q_{\ell j}^{(\text {out })} e^{q_{\ell \ell}^{(\text {out })} y} \ell_{i \ell}\left(t-I_{\ell j}^{(\text {out })}(y)-z\right) \delta\left(x-I_{\ell j}^{(o u t)}(y)-z\right) d y+\sigma(D) d z}_{\Theta(D)}, \\
& =\ell_{i j}(t-x) \mathcal{I}_{\left\{0<x-D / r_{j}^{(o u t)}<c D\right\}}+\Theta(D)
\end{aligned}
$$


Substituting the $\Theta(D)$ level description of the integral into $\ell_{i j}(t)$ we have

$$
\begin{aligned}
& \ell_{i j}(t)=\left(1+q_{i i}^{(i n)} \frac{D}{r_{i}^{(i n)}}\right) \int_{y=0}^{\frac{D}{r_{i}^{(i n)}}} g_{i j}\left(t-y, \frac{D}{r_{i}^{(i n)}}-y\right) d y+
\end{aligned}
$$

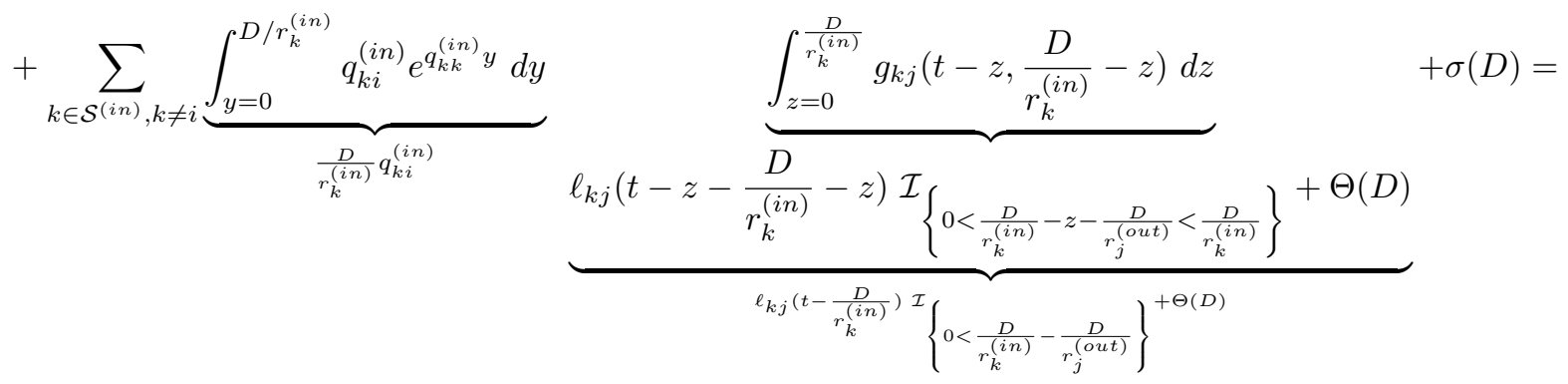

$$
\begin{aligned}
& =\int_{y=0}^{\frac{D}{r_{i}^{(i n)}}} g_{i j}\left(t-y, \frac{D}{r_{i}^{(i n)}}-y\right) d y+\sum_{k \in \mathcal{S}^{(i n)}} \frac{D}{r_{k}^{(i n)}} q_{k i}^{(i n)} \ell_{k j}\left(t-\frac{D}{r_{k}^{(i n)}}\right) \mathcal{I}_{\left\{0<\frac{D}{r_{k}^{(i n)}}-\frac{D}{r_{j}^{(\text {out })}}\right\}}+\sigma(D)
\end{aligned}
$$

from which

$$
\ell_{i j}(t)=\int_{y=0}^{\frac{D}{r_{i}^{(i n)}}} g_{i j}\left(t-y, \frac{D}{r_{i}^{(i n)}}-y\right) d y+\sum_{k: r_{j}^{(o u t)}>r_{k}^{(i n)}} \frac{D}{r_{k}^{(i n)}} q_{k i}^{(i n)} \ell_{k j}\left(t-\frac{D}{r_{k}^{(i n)}}\right)+\sigma(D) .
$$

In order to evaluate the remaining integral in (B.15) we need its more detailed, $\sigma(D)$ level description. It is obtained as follows

$$
\begin{aligned}
& g_{i j}(t, x)=\left(1+q_{j j}^{(\text {out })} \frac{D}{r_{j}^{(\text {out })}}\right) f_{i j}\left(t-\frac{D}{r_{j}^{(\text {out })}}, x-\frac{D}{r_{j}^{(\text {out })}}\right) \mathcal{I}_{\left\{x>D / r_{j}^{(\text {out })}\right\}^{+}} \\
& +\left(1+q_{j j}^{(\text {out })} \frac{D}{r_{j}^{(\text {out })}}\right) \ell_{i j}\left(t-\frac{D}{r_{j}^{(\text {out })}}\right) \delta\left(x-D / r_{j}^{(\text {out })}\right)+ \\
& \left.+\sum_{\ell \in \mathcal{S}^{(\text {out })}, \ell \neq j} q_{\ell j}^{\text {(out) }} \frac{D}{r_{\ell}^{(\text {out })}} f_{i \ell}\left(t-\frac{D}{r_{\ell}^{(\text {out })}}, x-\frac{D}{r_{\ell}^{(\text {out })}}\right) \mathcal{I}_{\left\{>\frac{D}{r_{\ell}^{(o u t)}}\right.}\right\}^{+} \\
& +\sum_{\ell \in \mathcal{S}(\text { out }), \ell \neq j} q_{\ell j}^{(\text {out })} \frac{D}{r_{\ell}^{(\text {out })}} \ell_{i \ell}\left(t-\frac{D}{r_{\ell}^{(\text {out })}}\right) \delta\left(x-\frac{D}{r_{\ell}^{(\text {out })}}\right) d y+\sigma(D) \\
& =f_{i j}\left(t-\frac{D}{r_{j}^{(\text {out })}}, x-\frac{D}{r_{j}^{(\text {out })}}\right) \mathcal{I}_{\left\{x>D / r_{j}^{(\text {out })}\right\}^{+}} \\
& +\ell_{i j}\left(t-\frac{D}{r_{j}^{(\text {out })}}\right) \delta\left(x-D / r_{j}^{(\text {out })}\right)+ \\
& \left.+\sum_{\ell \in \mathcal{S}^{\text {(out })}} q_{\ell j}^{(\text {out })} \frac{D}{r_{\ell}^{(\text {out })}} f_{i \ell}\left(t-\frac{D}{r_{\ell}^{(\text {out })}}, x-\frac{D}{r_{\ell}^{(\text {out })}}\right) \mathcal{I}_{\left\{x>\frac{D}{r_{\ell}^{(o u t)}}\right.}\right\}^{+} \\
& +\sum_{\ell \in \mathcal{S}^{\text {(out })}} q_{\ell j}^{(\text {out })} \frac{D}{r_{\ell}^{(\text {out })}} \ell_{i \ell}\left(t-\frac{D}{r_{\ell}^{(\text {out })}}\right) \delta\left(x-\frac{D}{r_{\ell}^{(\text {out })}}\right) d y+\sigma(D),
\end{aligned}
$$


Integrating the last expression we get

$$
\begin{aligned}
& \int_{y=0}^{\frac{D}{r_{i}^{(i n)}}} g_{i j}\left(t-y, \frac{D}{r_{i}^{(i n)}}-y\right) d y= \\
& \left.=\int_{y=0}^{\frac{D}{r_{i}^{(\text {in })}}} f_{i j}\left(t-y-\frac{D}{r_{j}^{(\text {out })}}, \frac{D}{r_{i}^{(\text {in })}}-y-\frac{D}{r_{j}^{(\text {out })}}\right) \mathcal{I}_{\left\{\frac{D}{r_{i}^{(\text {in })}}-y>D / r_{j}^{(\text {out })}\right.}\right\} d y+ \\
& +\int_{y=0}^{\frac{D}{r_{i}^{(\text {in })}}} \ell_{i j}\left(t-y-\frac{D}{r_{j}^{(\text {out })}}\right) \delta\left(\frac{D}{r_{i}^{(\text {in })}}-y-D / r_{j}^{(\text {out })}\right) d y+ \\
& +\sum_{\ell \in \mathcal{S}_{\text {(out })}} \underbrace{\int_{y=0}^{\frac{D}{r_{i}^{(i n)}}} q_{\ell j}^{(\text {out })} \frac{D}{r_{\ell}^{(\text {out })}} f_{i \ell}\left(t-y-\frac{D}{r_{\ell}^{(\text {out })}}, \frac{D}{r_{i}^{(\text {in })}}-y-\frac{D}{r_{\ell}^{(\text {out })}}\right) \mathcal{I}_{\left\{\frac{D}{r_{i}^{(i n)}}-y>\frac{D}{r_{\ell}^{(\text {out })}}\right\}} d y}_{\sigma(D)}+ \\
& +\sum_{\ell \in \mathcal{S}_{\text {(out })}} \int_{y=0}^{\frac{D}{r_{i}^{\text {(in) }}}} q_{\ell j}^{\text {(out })} \frac{D}{r_{\ell}^{(\text {out })}} \ell_{i \ell}\left(t-y-\frac{D}{r_{\ell}^{(\text {out })}}\right) \delta\left(\frac{D}{r_{i}^{(\text {in })}}-y-\frac{D}{r_{\ell}^{(\text {out })}}\right) d y+\sigma(D)
\end{aligned}
$$

for $1 / r_{i}^{(i n)}>1 / r_{j}^{(o u t)}$ (that is the drift is negative) it is

$$
\begin{aligned}
& =\int_{y=0}^{\frac{D}{r_{i}^{(\text {in })}}-\frac{D}{r_{j}^{(\text {out })}}} f_{i j}\left(t-y-\frac{D}{r_{j}^{(\text {out })}}, \frac{D}{r_{i}^{(\text {in })}}-y-\frac{D}{r_{j}^{(\text {out })}}\right) d y+\ell_{i j}\left(t-\frac{D}{r_{i}^{(\text {in })}}\right)+ \\
& +\sum_{\ell: r_{\ell}^{(\text {out })}>r_{i}^{(i n)}} q_{\ell j}^{(\text {out })} \frac{D}{r_{\ell}^{(\text {out })}} \ell_{i \ell}\left(t-\frac{D}{r_{i}^{(\text {in })}}\right)+\sigma(D)= \\
& =\left(\frac{D}{r_{i}^{(\text {in })}}-\frac{D}{r_{j}^{(\text {out })}}\right) f_{i j}\left(t-\frac{D}{r_{j}^{(\text {out })}}, 0\right)+\ell_{i j}\left(t-\frac{D}{r_{i}^{(\text {in })}}\right)+ \\
& +\sum_{\ell: r_{\ell}^{(\text {out })}>r_{i}^{(\text {in })}} q_{\ell j}^{(\text {out })} \frac{D}{r_{\ell}^{(\text {out })}} \ell_{i \ell}\left(t-\frac{D}{r_{i}^{(\text {in })}}\right)+\sigma(D) .
\end{aligned}
$$

Substituting this expression into (B.15) and expressing $f_{i j}(t)$ from $g_{i j}(t)$ based on (B.8) we get

$$
\begin{aligned}
\ell_{i j}(t) & =\left(\frac{D}{r_{i}^{(\text {in })}}-\frac{D}{r_{j}^{(\text {out })}}\right) g_{i j}\left(t-\frac{D}{r_{j}^{(\text {out })}}, 0\right)+\ell_{i j}\left(t-\frac{D}{r_{i}^{(\text {in })}}\right)+ \\
& +\sum_{\ell: r_{\ell}^{(\text {out })}>r_{i}^{(i n)}} q_{\ell j}^{(\text {out })} \frac{D}{r_{\ell}^{(\text {out })}} \ell_{i \ell}\left(t-\frac{D}{r_{i}^{(\text {in })}}\right)+ \\
& +\sum_{k: r_{j}^{(\text {out })}>r_{k}^{(i n)}} \frac{D}{r_{k}^{(\text {in })}} q_{k i}^{(\text {in })} \ell_{k j}\left(t-\frac{D}{r_{k}^{(\text {in })}}\right)+\sigma(D)
\end{aligned}
$$

Extracting $\ell_{i j}\left(t-\frac{D}{r_{i}^{(i n)}}\right)$, dividing by $D$ gives

$$
\begin{aligned}
& \frac{\ell_{i j}(t)-\ell_{i j}\left(t-\frac{D}{r_{i}^{(\text {in })}}\right)}{D}=\left(\frac{1}{r_{i}^{(\text {in })}}-\frac{1}{r_{j}^{(\text {out })}}\right) g_{i j}\left(t-\frac{D}{r_{j}^{(\text {out })}}, 0\right)+ \\
& +\sum_{\ell: r_{\ell}^{(\text {out })}>r_{i}^{(\text {in })}} \frac{q_{\ell j}^{(\text {out })}}{r_{\ell}^{(\text {out })}} \ell_{i \ell}\left(t-\frac{D}{r_{i}^{(\text {in })}}\right)+\sum_{k: r_{j}^{(\text {out })}>r_{k}^{(i n)}} \frac{q_{k i}^{(\text {in })}}{r_{k}^{(i n)}} \ell_{k j}\left(t-\frac{D}{r_{k}^{(\text {in })}}\right)+\frac{\sigma(D)}{D},
\end{aligned}
$$




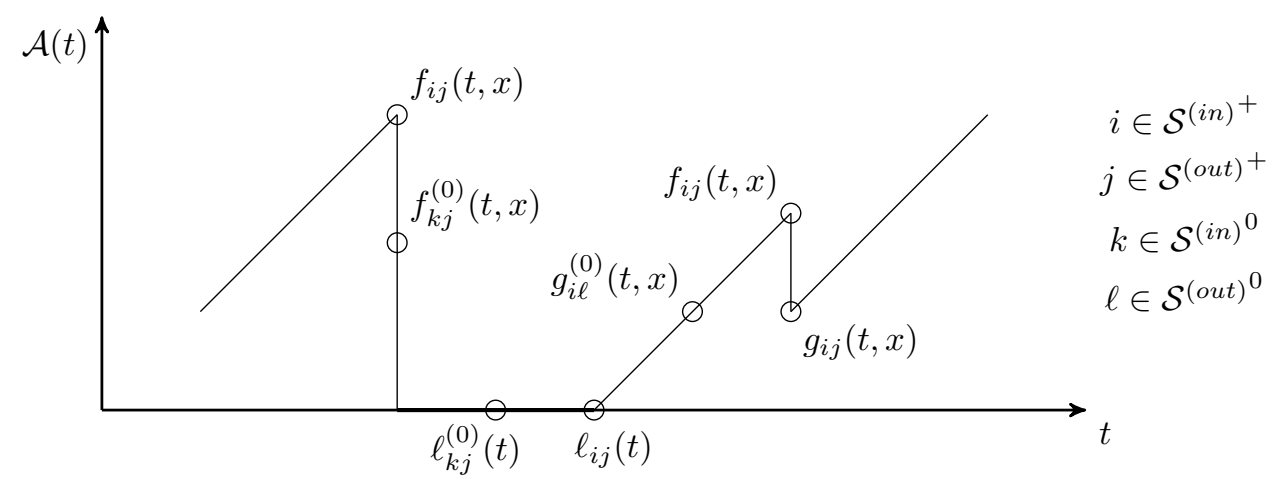

Figure B.2: Age process with zero rate

which, when taking the $D \rightarrow 0$ and the $t \rightarrow \infty$ limits and assuming that the stationary limit exist, gives

$$
\left(\frac{1}{r_{i}^{(\text {out })}}-\frac{1}{r_{j}^{(\text {in })}}\right) g_{i j}(0)=\sum_{\ell: r_{\ell}^{(o u t)}>r_{i}^{(i n)}} \frac{q_{\ell j}^{(\text {out })}}{r_{\ell}^{(\text {out })}} \ell_{i \ell}+\sum_{k: r_{j}^{(\text {out })}>r_{k}^{(i n)}} \frac{q_{k i}^{(i n)}}{r_{k}^{(i n)}} \ell_{k j},
$$

Assuming that the stationary limit exist, $\lim _{t \rightarrow \infty} g_{i j}(t, x)=g_{i j}(x)$, the vector representation of (B.16) is

$$
g_{-}(0)\left(-\mathbf{R}^{(\text {in })^{-1}} \otimes \mathbf{I}+\mathbf{I} \otimes \mathbf{R}^{(\text {out })^{-1}}\right)_{-}=\ell_{-}\left(\mathbf{R}^{(\text {in })^{-1}} \mathbf{Q}^{(\text {in })} \otimes \mathbf{I}+\mathbf{I} \otimes \mathbf{R}^{(\text {out })^{-1}} \mathbf{Q}^{(\text {out })}\right)_{-} .
$$

Next we relate the resulted differential and boundary equations with the ones for the special fluid model characterized by $\left(\mathbf{R}, \tilde{\mathbf{Q}}, \tilde{\mathbf{Q}}^{(\mathbf{0})}=\tilde{\mathbf{Q}}\right)$.

Proof. Introducing $\tilde{g}(x)=g(x)\left(\mathbf{R}^{(\text {in })^{-1}} \otimes \mathbf{R}^{(\text {out })^{-1}}\right)$ and $\tilde{\ell}=\ell\left(\mathbf{R}^{(\text {in })^{-1}} \otimes \mathbf{R}^{(\text {out })^{-1}}\right)$ from (B.12) and (B.17) we have

$$
\begin{aligned}
& \frac{\partial}{\partial x} \tilde{g}(x) \underbrace{\left(\mathbf{R}^{(\text {in })} \otimes \mathbf{I}-\mathbf{I} \otimes \mathbf{R}^{(\text {out })}\right)}_{\mathbf{R}}=\tilde{g}(x) \underbrace{\left(\mathbf{R}^{(\text {in })} \otimes \mathbf{Q}^{(\text {out })}+\mathbf{Q}^{(\text {in })} \otimes \mathbf{R}^{(\text {out })}\right)}_{\tilde{\mathbf{Q}}}, \\
& \tilde{g}_{-}(0) \underbrace{\left(\mathbf{R}^{(\text {in })} \otimes \mathbf{I}-\mathbf{I} \otimes \mathbf{R}^{(\text {out })}\right)_{-}}_{\mathbf{R}_{-}}=\tilde{\ell}_{-}^{\left(\mathbf{R}^{(\text {in })} \otimes \mathbf{Q}^{(\text {out })}+\mathbf{Q}^{(\text {in })} \otimes \mathbf{R}^{(\text {out })}\right)} \underbrace{}_{\tilde{\mathbf{Q}}_{--}} .
\end{aligned}
$$

Which means that $\tilde{g}(x)=\tilde{\pi}(x)$ if the same normalizing conditions are in place, because they satisfies the same set of equations with unique solution. Finally equation (52) is obtained from $g(x)=\tilde{\pi}(x)\left(\mathbf{R}^{(\mathbf{i n})} \otimes \mathbf{R}^{(\text {out })}\right) \frac{1}{\lambda \mu}$, where the last scaling constant accounts for the proper normalization of $g(x)$.

Appendix B.2. The Proof of Theorem 5 with zero $r^{(i n)}$ or $r^{(\text {out })}$ rates

PROOF. The proof is built on a similar analysis of the drop service process. In this part we do not repeat the derivations, but only emphasize the differences compared to the nonzero case. 
In this case we need the following probabilities

$$
\begin{aligned}
& f_{i j}(t, x)=P\left(\mathcal{Z}^{(i n)}(t-x)=i, \mathcal{Z}^{(\text {out })}(t)=j, \mathcal{A}\left(t^{+}\right)=x \mid \text { drop arrival at } t-x\right), \quad x>0, i \in \mathcal{S}^{(i n)^{+}}, \\
& f_{i j}^{0}(t, x)=P\left(\mathcal{Z}^{(i n)}(t-x)=i, \mathcal{Z}^{(\text {out })}(t)=j, \mathcal{A}(t)=x \mid \text { drop departure at } t\right), \quad x>0, i \in \mathcal{S}^{(\text {in })^{0}}, \\
& \left.g_{i j}(t, x)=P\left(\mathcal{Z}^{(i n)}(t-x)=i, \mathcal{Z}^{(\text {out })}(t)=j, \mathcal{A}\left(t^{-}\right)=x \mid \text { drop arrival at } t\right)\right), \quad x>0, j \in \mathcal{S}^{(\text {out })^{+}}, \\
& g_{i j}^{0}(t, x)=P\left(\mathcal{Z}^{(i n)}(t-x)=i, \mathcal{Z}^{(\text {out })}(t)=j, \mathcal{A}(t)=x \mid \text { no drop departure at } t\right), \quad x>0, j \in \mathcal{S}^{(\text {out })^{0}} .
\end{aligned}
$$

It is very important to note that the discontinuity points of the age process are associated with such states of the input and output Markov chain where the input and output rates are strictly positive, since drop arrival and service can happen only in states with positive input and output rates. Consequently states with zero rates can show up only in between the corner points of the discontinuities of the age process (see Figure B.2).

To characterize the age process when the buffer is empty we further define

$$
\begin{aligned}
& \ell_{i j}(t)=P\left(\mathcal{Z}^{(i n)}(t)=i, \mathcal{Z}^{(\text {out })}(t)=j, \mathcal{A}(t)=0\right), i \in \mathcal{S}^{(i n)^{+}}, \\
& \ell_{i j}^{0}(t)=P\left(\mathcal{Z}^{(i n)}(t)=i, \mathcal{Z}^{(\text {out })}(t)=j, \mathcal{A}(t)=0\right), i \in \mathcal{S}^{(i n)^{0}} .
\end{aligned}
$$

With zero input rate (B.4) becomes

$$
\begin{aligned}
f_{i j}(t, x)= & \left(1+q_{i i}^{(i n)} \frac{D}{r_{i}^{(i n)}}\right) g_{i j}\left(t, x+\frac{D}{r_{i}^{(i n)}}\right)+\sigma(D)+ \\
& +\sum_{k \in \mathcal{S}^{(i n)+}{ }_{, k \neq i}} q_{k i}^{(i n)} \frac{D}{r_{k}^{(i n)}} g_{k j}(t, x+\Theta(D))+\sum_{\left.k \in \mathcal{S}^{(i n)}\right)^{0}} q_{k i}^{(i n)} D f_{k j}^{0}(t, x+\Theta(D)) .
\end{aligned}
$$

Similarly for $f_{i j}^{0}(t, x)$ we have

$$
\begin{aligned}
f_{i j}^{0}(t, x)= & \left(1+q_{i i}^{(i n)} D\right) f_{i j}^{0}(t, x+D)+\sigma(D)+ \\
& +\sum_{k \in \mathcal{S}^{(i n)}+} q_{k i}^{(i n)} \frac{D}{r_{k}^{(i n)}} g_{k j}(t, x+\Theta(D))+\sum_{k \in \mathcal{S}^{(i n)^{0}}, k \neq i} q_{k i}^{(i n)} D f_{k j}^{0}(t, x+\Theta(D)) .
\end{aligned}
$$

With zero input rate and $x>\max _{j} r_{j}^{(\text {out })} D($ B.7) takes the form

$$
\begin{aligned}
g_{i j}(t, x)= & \left(1+q_{j j}^{(\text {out })} \frac{D}{r_{j}^{(\text {out })}}\right) f_{i j}\left(t-\frac{D}{r_{j}^{(\text {out })}}, x-\frac{D}{r_{j}^{(\text {out })}}\right)+\sigma(D)+ \\
& +\sum_{\ell \in \mathcal{S}^{(\text {out })+}, \ell \neq j} q_{\ell j}^{(\text {out })} \frac{D}{r_{\ell}^{(\text {out })}} f_{i \ell}(t-\Theta(D), x-\Theta(D))+ \\
& +\sum_{\ell \in \mathcal{S}^{(\text {out })} 0} q_{\ell j}^{(\text {out })} D g_{i \ell}^{0}(t-\Theta(D), x-\Theta(D))
\end{aligned}
$$

and for $g_{i j}^{0}(t, x)$ we have

$$
\begin{aligned}
g_{i j}^{0}(t, x)= & \left(1+q_{j j}^{\text {(out })} D\right) g_{i j}^{0}(t-D, x-D)+\sigma(D)+ \\
& +\sum_{\ell \in \mathcal{S}_{(\text {out })+}} q_{\ell j}^{(\text {out })} \frac{D}{r_{\ell}^{(\text {out })}} f_{i \ell}(t-\Theta(D), x-\Theta(D))+ \\
& +\sum_{\ell \in \mathcal{S}^{(\text {out })^{0}}, \ell \neq j} q_{\ell j}^{(\text {out })} D g_{i \ell}^{0}(t-D, x-D) .
\end{aligned}
$$


With zero input rate (B.20)-(B.23) can be rewritten as

$$
\begin{aligned}
& f_{i j}(t, x)=g_{i j}(t, x)+\frac{D}{r_{i}^{(i n)}} \frac{\partial}{\partial x} g_{i j}(t, x)+\sigma(D)+ \\
& +\sum_{k \in \mathcal{S}^{(i n)+}} q_{k i}^{(i n)} \frac{D}{r_{k}^{(i n)}} g_{k j}(t, x)+\sum_{\left.k \in \mathcal{S}^{(i n)}\right)^{0}} q_{k i}^{(i n)} D f_{k j}^{0}(t, x) . \\
& f_{i j}^{0}(t, x)=f_{i j}^{0}(t, x)+D \frac{\partial}{\partial x} f_{i j}^{0}(t, x)+\sigma(D)+ \\
& +\sum_{k \in \mathcal{S}^{(i n)+}} q_{k i}^{(i n)} \frac{D}{r_{k}^{(i n)}} g_{k j}(t, x)+\sum_{k \in \mathcal{S}^{(i n) 0}} q_{k i}^{(i n)} D f_{k j}^{0}(t, x) . \\
& g_{i j}(t, x)=f_{i j}(t, x)-\frac{D}{r_{j}^{(o u t)}} \frac{\partial}{\partial t} f_{i j}(t, x)-\frac{D}{r_{j}^{(o u t)}} \frac{\partial}{\partial x} f_{i j}(t, x)+\sigma(D)+ \\
& +\sum_{\ell \in \mathcal{S}^{(\text {out })}+} q_{\ell j}^{(\text {out })} \frac{D}{r_{\ell}^{(\text {out })}} f_{i \ell}(t, x)+\sum_{\ell \in \mathcal{S}^{(o u t)} 0} q_{\ell j}^{(\text {out })} D g_{i \ell}^{0}(t, x) . \\
& g_{i j}^{0}(t, x)=g_{i j}^{0}(t, x)-D \frac{\partial}{\partial t} g_{i j}^{0}(t, x)-D \frac{\partial}{\partial x} g_{i j}^{0}(t, x)+\sigma(D)+ \\
& +\sum_{\ell \in \mathcal{S}^{(\text {out })}+} q_{\ell j}^{(\text {out })} \frac{D}{r_{\ell}^{(\text {out })}} f_{i \ell}(t, x)+\sum_{\ell \in \mathcal{S}^{(\text {out })} 0} q_{\ell j}^{(\text {out })} D g_{i \ell}^{0}(t, x) .
\end{aligned}
$$

The matrix form of (B.24)-(B.27) are as follows.

$$
\begin{aligned}
& f(t, x)=g(t, x)+\frac{\partial}{\partial x} g(t, x)\left(\mathbf{R}_{+}^{(\mathbf{i n})^{-1}} \otimes \mathbf{I}\right) D+\sigma(D)+ \\
& +g(t, x)\left(\mathbf{R}_{+}^{(\mathbf{i n})^{-1}} \mathbf{Q}_{++}^{(\mathbf{i n})} \otimes \mathbf{I}\right) D+f^{0}(t, x)\left(\mathbf{Q}_{\mathbf{0}+}^{(\mathbf{i n})} \otimes \mathbf{I}\right) D . \\
& f^{0}(t, x)=f^{0}(t, x)+\frac{\partial}{\partial x} f^{0}(t, x) D+\sigma(D)+ \\
& +g(t, x)\left(\mathbf{R}_{+}^{(\mathbf{i n})^{-1}} \mathbf{Q}_{\mathbf{0}+}^{(\mathbf{i n})} \otimes \mathbf{I}\right) D+f^{0}(t, x)\left(\mathbf{Q}_{\mathbf{0 0}}^{(\mathbf{i n})} \otimes \mathbf{I}\right) D . \\
& \left.g(t, x)=f(t, x)-\frac{\partial}{\partial t} f(t, x)\left(\mathbf{I} \otimes \mathbf{R}_{+}^{(\text {out })^{-1}}\right) D-\frac{\partial}{\partial x} f(t, x)\left(\mathbf{I} \otimes \mathbf{R}_{+}^{(\text {out }}\right)^{-1}\right) D+\sigma(D)+ \\
& +f(t, x)\left(\mathbf{I} \otimes \mathbf{R}_{+}^{(\text {out })^{-1}} \mathbf{Q}_{++}^{(\text {out })}\right) D+g^{0}(t, x)\left(\mathbf{I} \otimes \mathbf{Q}_{\mathbf{0}+}^{(\text {out })}\right) D . \\
& g^{0}(t, x)=g^{0}(t, x)-\frac{\partial}{\partial t} g^{0}(t, x) D-\frac{\partial}{\partial x} g^{0}(t, x) D+\sigma(D)+ \\
& +f(t, x)\left(\mathbf{I} \otimes \mathbf{R}_{+}^{(\text {out })^{-1}} \mathbf{Q}_{+\mathbf{0}}^{(\text {out })}\right) D+g^{0}(t, x)\left(\mathbf{I} \otimes \mathbf{Q}_{\mathbf{0 0}}^{(\text {out })}\right) D .
\end{aligned}
$$

Dividing the equations by $D$ letting $D \rightarrow 0, t \rightarrow \infty$, and substituting (B.28) into (B.30) and (B.31) gives.

$$
-\frac{\partial}{\partial x} f^{0}(x)=g(x)\left(\mathbf{R}_{+}^{(\mathbf{i n})^{-1}} \mathbf{Q}_{+\mathbf{0}}^{(\mathbf{i n})} \otimes \mathbf{I}\right)+f^{0}(x)\left(\mathbf{Q}_{\mathbf{0 0}}^{(\mathbf{i n})} \otimes \mathbf{I}\right) .
$$




$$
\begin{gathered}
0=\frac{\partial}{\partial x} g(x)\left(\mathbf{R}_{+}^{(\text {in })^{-1}} \otimes \mathbf{I}\right)+g(x)\left(\mathbf{R}_{+}^{(\text {in })^{-1}} \mathbf{Q}_{++}^{\text {(in })} \otimes \mathbf{I}\right)+f^{0}(x)\left(\mathbf{Q}_{\mathbf{0}+}^{(\text {in })} \otimes \mathbf{I}\right) \\
-\frac{\partial}{\partial x} g(x)\left(\mathbf{I} \otimes \mathbf{R}_{+}^{(\text {out })^{-1}}\right)+g(x)\left(\mathbf{I} \otimes \mathbf{R}_{+}^{(\text {out })^{-1}} \mathbf{Q}_{++}^{(\text {out })}\right)+g^{0}(x)\left(\mathbf{I} \otimes \mathbf{Q}_{\mathbf{0}+}^{(\text {out })}\right) . \\
\frac{\partial}{\partial x} g^{0}(x)=g(x)\left(\mathbf{I} \otimes \mathbf{R}_{+}^{(\text {out })^{-1}} \mathbf{Q}_{+\mathbf{0}}^{(\text {out })}\right)+g^{0}(x)\left(\mathbf{I} \otimes \mathbf{Q}_{\mathbf{0 0}}^{(\text {out })}\right) .
\end{gathered}
$$

Similar to the non-zero case next we relate the resulted differential and boundary equations with the ones for the special fluid model characterized by $\left(\mathbf{R}, \tilde{\mathbf{Q}}, \tilde{\mathbf{Q}}^{(\mathbf{0})}=\tilde{\mathbf{Q}}\right)$.

Proof. Considering the $\mathbf{R}^{(\mathbf{i n})}=\left(\begin{array}{cc}\mathbf{R}_{+}^{(\mathbf{i n})} & \mathbf{0} \\ \mathbf{0} & \mathbf{0}\end{array}\right)$ and the $\mathbf{R}^{(\text {out })}=\left(\begin{array}{cc}\mathbf{R}_{+}^{(\text {out })} & \mathbf{0} \\ \mathbf{0} & \mathbf{0}\end{array}\right)$ state partitioning of the rate matrices and the associated $\tilde{\pi}(x)=\left[\tilde{\pi}_{++}(x), \tilde{\pi}_{+0}(x), \tilde{\pi}_{0+}(x), \tilde{\pi}_{00}(x)\right]$ vector partitioning the $\frac{d}{d t} \tilde{\pi}(x) \mathbf{R}=$ $\tilde{\pi}(x) \tilde{\mathbf{Q}}$ differential equation has the following block structure.

$$
\begin{aligned}
\frac{d}{d t} \tilde{\pi}_{++}(x)\left(\mathbf{R}_{+}^{(\mathbf{i n})} \otimes \mathbf{I}-\mathbf{I} \otimes \mathbf{R}_{+}^{(\text {out })}\right) & =\tilde{\pi}_{++}(x)\left(\mathbf{R}_{+}^{(\text {in) }} \otimes \mathbf{Q}_{++}^{(\text {out })}+\mathbf{Q}_{++}^{(\mathbf{i n})} \otimes \mathbf{R}_{+}^{(\text {out })}\right) \\
& +\tilde{\pi}_{0+}(x)\left(\mathbf{Q}_{\mathbf{0}+}^{(\mathbf{i n})} \otimes \mathbf{R}_{+}^{(\text {out })}\right)+\tilde{\pi}_{+0}(x)\left(\mathbf{R}_{+}^{(\text {in })} \otimes \mathbf{Q}_{\mathbf{0}+}^{(\text {out })}\right) \\
\frac{d}{d t} \tilde{\pi}_{+0}(x)\left(\mathbf{R}_{+}^{(\mathbf{i n})} \otimes \mathbf{I}\right)= & \tilde{\pi}_{++}(x)\left(\mathbf{R}_{+}^{(\text {in })} \otimes \mathbf{Q}_{+\mathbf{0}}^{(\text {out })}\right)+\tilde{\pi}_{+0}(x)\left(\mathbf{R}_{+}^{(\mathbf{i n})} \otimes \mathbf{Q}_{\mathbf{0 0}}^{(\text {out })}\right) \\
-\frac{d}{d t} \tilde{\pi}_{0+}(x)\left(\mathbf{I} \otimes \mathbf{R}_{+}^{(\text {out })}\right)= & \tilde{\pi}_{++}(x)\left(\mathbf{Q}_{+\mathbf{0}}^{(\text {in })} \otimes \mathbf{R}_{+}^{(\text {out })}\right)+\tilde{\pi}_{0+}(x)\left(\mathbf{Q}_{\mathbf{0 0}}^{(\text {in })} \otimes \mathbf{R}_{+}^{(\text {out })}\right)
\end{aligned}
$$

On the other hand, introducing $\tilde{g}(x)=g(x)\left(R_{+}^{(i n)^{-1}} \otimes R_{+}^{(o u t)^{-1}}\right), \tilde{g}^{0}(x)=g^{0}(x)\left(R_{+}^{(i n)^{-1}} \otimes I\right)$ and $\tilde{f}^{0}(x)=$ $\left.f^{0}(x)\left(I \otimes R_{+}^{(o u t)}\right)^{-1}\right)$ from (B.32)-(B.34) we obtain equations (B.35)-(B.37) with $\tilde{\pi}_{++}(x)=\tilde{g}(x), \tilde{\pi}_{+0}(x)=$ $\tilde{f}^{0}(x)$, with $\tilde{\pi}_{0+}(x)=\tilde{g}^{0}(x)$.

Using the same approach it is possible to prove that the boundary equations given by (B.19) are valid in case of zero $r^{(i n)}$ or $r^{(\text {out })}$ rates as well.

\section{Appendix C. Proof of Theorem 7}

Proof. The proof is very similar to the proof of Theorem 5. The only difference is that instead of (B.13) we can express $\ell_{i j}(t)$ a bit differently as

$$
\begin{aligned}
\ell_{i j}(t) & =\left(1+q_{i i}^{(i n)} \frac{D}{r_{i}^{(i n)}}\right) \times \\
& \times \int_{y=0}^{\frac{D}{r_{i}^{(i n)}}}\left[\left(1+q_{j j}^{(\text {out })} y\right) g_{i j}\left(t-y, \frac{D}{r_{i}^{(i n)}}-y\right)+\sum_{m \in \mathcal{S}^{(\text {out })}, m \neq j} q_{m j}^{(\text {out })} y g_{i m}\left(t-y, \frac{D}{r_{i}^{(\text {in })}}-y\right)\right] d y+ \\
& +\sum_{k \in \mathcal{S}^{(i n)}, k \neq i} \int_{y=0}^{D / r_{k}^{(i n)}} q_{k i}^{(i n)} e^{q_{k k}^{(i n)} y} d y \int_{z=0}^{\frac{D}{r_{k}^{(i n)}}}\left[\left(1+q_{j j}^{(\text {out })} z\right) g_{k j}\left(t-z, \frac{D}{r_{k}^{(i n)}}-z\right)+\right. \\
& \left.+\sum_{m \in \mathcal{S}^{(\text {out })}, m \neq j} q_{m j}^{(\text {out })} z g_{k m}\left(t-z, \frac{D}{r_{k}^{(i n)}}-z\right)\right] d z+\sigma(D) .
\end{aligned}
$$

Applying the same steps as in Appendix Appendix B establishes the theorem. 